\title{
Vapor Pressures, Densities, and PC-SAFT Parameters for 11 Bio-compounds
}

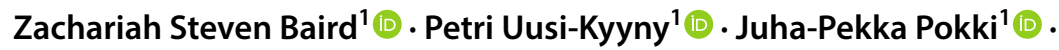 Emilie Pedegert ${ }^{1} \cdot$ Ville Alopaeus $^{1}{ }^{\mathbb{D}}$}

Received: 13 March 2019 / Accepted: 11 October 2019 / Published online: 6 November 2019

(c) The Author(s) 2019

\begin{abstract}
One major sustainable development goal is to produce chemicals and fuels from renewable resources, such as biomass, rather than from fossil fuels. A key part of this development is data on the properties of chemicals that appear in this bio-based supply chain. Many of the chemicals have yet to be studied thoroughly, and data on their properties is lacking. Here, we present new experimental data on the properties of 11 bio-compounds, along with PC-SAFT parameters for modeling their properties. The measured data includes vapor pressures, compressed densities, and refractive indexes. The 11 bio-compounds are tetrahydrofuran, 2-pentanone, furfural, 2-methoxy-4-methylphenol, 2-methylfuran, dihydrolevoglucosenone, cyclopentyl methyl ether, 2-sec-butylphenol, levoglucosenone, $\gamma$-valerolactone, and 2,6-dimethoxyphenol.
\end{abstract}

Keywords Bio-compounds · Density · PC-SAFT $\cdot$ Thermodynamic properties · Vapor pressure

\section{Introduction}

The world is increasingly searching for sustainable substitutes to replace fossil fuels. Biomass from plants is one large resource that could be converted into fuels and chemicals [1]. To accomplish this goal, some have proposed the concept of a biorefinery, which would take raw materials such as biomass and convert them into valuable products needed in modern society [2]. Similar to the current petroleum-based supply chain, a bio-based supply chain would involve hundreds of different chemicals, whether as intermediates or final products $[1,2]$.

Petri Uusi-Kyyny

petri.uusi-kyyny@aalto.fi

1 Department of Chemical and Metallurgical Engineering, Aalto University, P.O. Box 16100, 00076 Espoo, Finland 
Chemicals relevant to the petroleum industry have been thoroughly studied over the past century, but for many bio-based chemicals there is little, if any, data about their properties. Key thermodynamic and physical properties of bio-compounds will need to be determined in order to produce bio-based chemicals. In this study we measured the vapor pressures, densities and refractive indexes of 11 such bio-compounds.

Several of these bio-compounds show up as intermediates or platform chemicals that could be further converted into a variety of products [2]. Many of the chemicals are, or could be, useful products. Dihydrolevoglucosenone is a potential bio-based alternative for dipolar aprotic solvents, such as $\mathrm{N}$-methyl-2-pyrrolidone and dimethylformamide [3,4]. 2,6-dimethoxyphenol has a smoky aroma and is an ingredient in artificial smoke-flavoring products [5, 6]. It, and its derivatives, could also be used for producing renewable phenolic resins [7]. 2-methoxy4-methylphenol is used as a flavoring [8]. 2-sec-Butylphenol has been successfully tested as a solvent for furfural extraction in the so-called biphasic reactor concept, and it may be possible to produce it from lignin [9]. It has also been proposed that alkylphenols, such as 2-sec-butylphenol, be used as a solvent in biofuel production to increase the effectiveness of the process [10]. $\gamma$-valerolactone can be used as a fuel and has also been identified as a promising platform chemical that can be used to produce a variety of other chemicals [11-14]. Tetrahydrofuran is used as a solvent and in producing some polymers [15, 16]. 2-methylfuran has received attention as a potential substitute for gasoline due to its impressive combustion performance [17]. Cyclopentyl methyl ether is used as a solvent, and it has been shown to be a promising solvent for extracting compounds from the aqueous streams present in bio-refineries [18, 19]. Furfural can be used as a selective solvent and is a platform chemical that can be processed into other products [20].

Many of these compounds can also be produced from one another. For instance, dihydrolevoglucosenone is produced via hydrogenation of levoglucosenone, which itself is produced from the sugar levoglucosan that is formed in the pyrolysis of lignocellulosic biomass [21]. In addition, there are reactions for converting between 2-methylfuran, tetrahydrofuran, furfural, and 2-pentanone [2, 22]. 1,3-dimethoxy-2-hydroxybenzene and 2-methoxy-4-methylphenol have been found in plants and in pyrolysis oil [23].

For 6 (2-methoxy-4-methylphenol, 2-sec-butylphenol, 2,6-dimethoxyphenol, cyclopentyl methyl ether, dihydrolevoglucosenone, levoglucosenone) of the 11 compounds there is only a small amount of data, if any at all. For 5 bio-compounds (2-methylfuran, 2-pentanone, furfural, tetrahydrofuran, $\gamma$-valerolactone) large amounts of data can be found in the literature. However, even for these 5 compounds the data presented here extends beyond the range covered in the literature. For 7 of the bio-compounds (2-methoxy-4-methylphenol, 2-methylfuran, 2-pentanone, cyclopentyl methyl ether, dihydrolevoglucosenone, furfural, tetrahydrofuran) we measured compressed densities at higher pressures (up to 12 or $16 \mathrm{MPa}$ ), and for many of the compounds there was no reliable data available at higher pressures. We have placed a file containing both the literature data we found and our experimental data in a repository at the Open Science Framework, 
and this file can be obtained at (https://osf.io/u9amn/). An overview of the measurements made in this work is presented in Table 1.

\section{Methods}

\subsection{Chemicals}

Information about the chemicals used in this study is presented in Tables 2 and 3. The structures of the chemicals are presented in Fig. 1 [24]. The purities were measured using gas chromatography with a flame ionization detector. For most of the chemicals the water content was also measured using a DL38 Karl Fischer Titrator (Mettler Toledo). The purity was calculated by taking the relative peak area from

Table 1 Overview of the measurements made in this work

\begin{tabular}{|c|c|c|c|}
\hline Name & Measured property & $\begin{array}{l}\text { Measurement } \\
\text { temperature, } \mathrm{K}\end{array}$ & Measurement pressure, $\mathrm{MPa}$ \\
\hline \multirow[t]{3}{*}{ 2-Methoxy-4-methylphenol } & Density, liquid & $293.15-473.15$ & $0.12-11.85$ \\
\hline & Vapor pressure, liquid & $298.22-403.2$ & $9.3 \mathrm{E}-6-5.3 \mathrm{E}-3$ \\
\hline & Refractive index, liquid & $293.15-343.15$ & 0.1 \\
\hline \multirow[t]{2}{*}{ 2-Methylfuran } & Density, liquid & $293.14-473.16$ & $0.09-11.85$ \\
\hline & Refractive index, liquid & 293.15 & 0.1 \\
\hline \multirow[t]{2}{*}{ 2-Pentanone } & Density, liquid & $293.15-473.16$ & $0.09-11.85$ \\
\hline & Refractive index, liquid & 293.15 & 0.1 \\
\hline \multirow[t]{3}{*}{ 2-sec-Butylphenol } & Density, liquid & $293.15-473.16$ & 0.101 \\
\hline & Vapor pressure, liquid & $298.21-403.25$ & $4.7 \mathrm{E}-6-4.6 \mathrm{E}-3$ \\
\hline & refractive index, liquid & $293.15-343.15$ & 0.1 \\
\hline \multirow[t]{3}{*}{ 2,6-Dimethoxyphenol } & Density, liquid & $333.16-383.16$ & 0.1 \\
\hline & Vapor pressure, liquid & $333.28-413.16$ & $1.4 \mathrm{E}-5-1.9 \mathrm{E}-3$ \\
\hline & Refractive index, liquid & $328.15-343.15$ & 0.1 \\
\hline Cyclopentyl methyl ether & Density, liquid & $293.14-473.15$ & $0.09-11.85$ \\
\hline \multirow[t]{3}{*}{ Dihydrolevoglucosenone } & Density, liquid & $293.15-423.16$ & $0.07-15.77$ \\
\hline & Vapor pressure, liquid & $298.26-403.16$ & $1.4 \mathrm{E}-5-5.2 \mathrm{E}-3$ \\
\hline & Refractive index, liquid & $293.15-343.15$ & 0.1 \\
\hline Furfural & Density, liquid & $293.15-448.15$ & $0.09-11.9$ \\
\hline \multirow[t]{3}{*}{ Levoglucosenone } & Density, liquid & $293.15-363.15$ & 0.1 \\
\hline & Vapor pressure, liquid & $298.26-403.3$ & $6.2 \mathrm{E}-6-3.5 \mathrm{E}-3$ \\
\hline & Refractive index, liquid & $293.15-343.15$ & 0.1 \\
\hline \multirow[t]{2}{*}{ Tetrahydrofuran } & Density, liquid & $293.14-473.15$ & $0.1-11.85$ \\
\hline & Refractive index, liquid & 293.15 & 0.1 \\
\hline \multirow[t]{2}{*}{$\gamma$-Valerolactone } & Vapor pressure, Liquid & $298.23-403.15$ & $4.4 \mathrm{E}-5-9.9 \mathrm{E}-3$ \\
\hline & Refractive index, liquid & $293.15-333.15$ & 0.1 \\
\hline
\end{tabular}




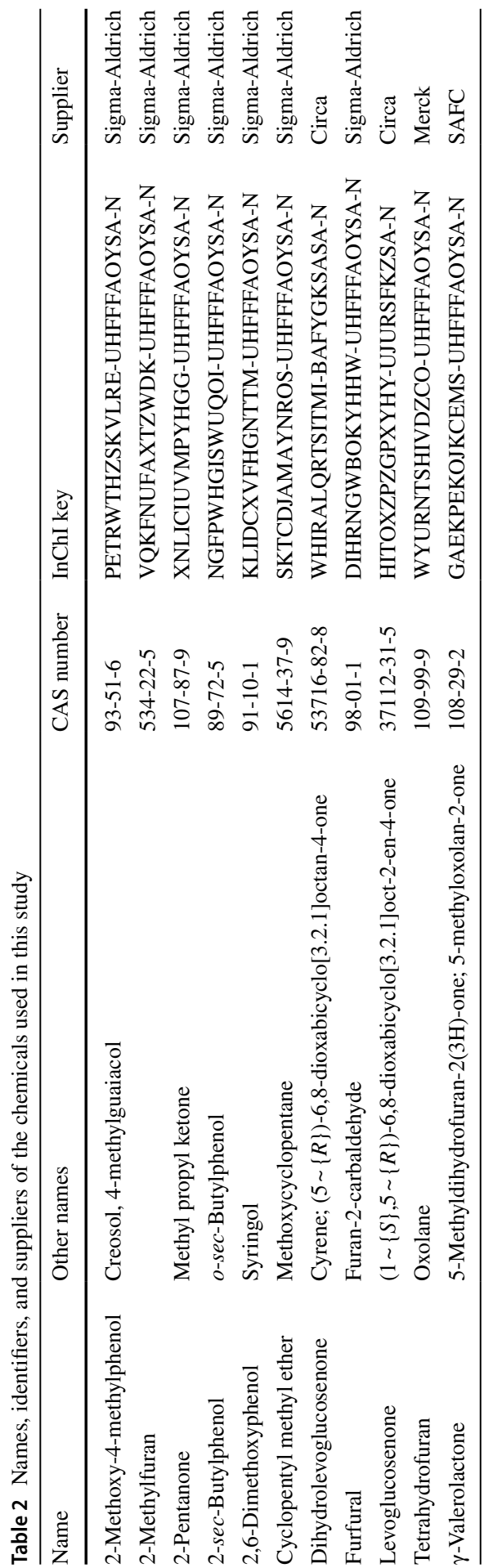


Table 3 Purities of the chemicals used in this study

\begin{tabular}{lllll}
\hline Name & Purification method & $\begin{array}{l}\text { Water con- } \\
\text { tent (wt })\end{array}$ & Purity (wt\%) & $\begin{array}{l}\text { Refractive index } \\
\text { nD (at 293 K) }\end{array}$ \\
\hline 2-Methoxy-4-methylphenol & & 0.39 & 99.4 & 1.5373 \\
2-Methylfuran & Distillation & 0.015 & 99.9 & 1.4332 \\
2-Pentanone & & 0.05 & 99.8 & 1.3903 \\
2-sec-Butylphenol & Vacuum distillation & & 99.9 & 1.5228 \\
2,6-Dimethoxyphenol & & & 99.7 & \\
Cyclopentyl methyl ether & & 0.0027 & 100.0 & 1.4732 \\
Dihydrolevoglucosenone & & 0.045 & 99.8 & \\
Furfural & Vacuum distillation & 0.013 & 99.8 & 1.5064 \\
Levoglucosenone & & & 96.2 & 1.5065 \\
Levoglucosenone & Vacuum distillation & 0.25 & 98.7 & 1.4073 \\
Tetrahydrofuran & & 0.033 & 99.9 & 1.4333 \\
$\gamma$-Valerolactone & Vacuum distillation & & 99.5 & \\
\hline
\end{tabular}

${ }^{\mathrm{a}}$ Atmospheric pressure $0.10 \pm 0.01 \mathrm{MPa}$

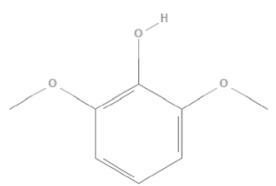

2,6-dimethoxyphenol

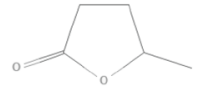

ү-valerolactone

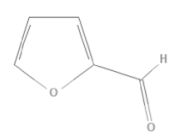

furfural
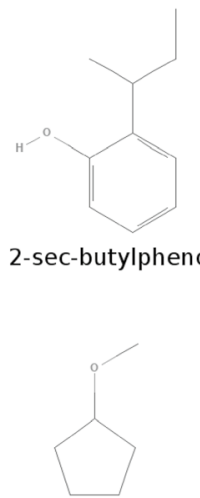

cyclopentyl methyl ether

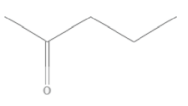

2-pentanone

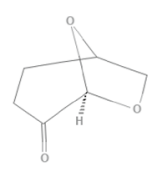

dihydrolevoglucosenone

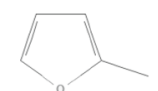

2-methylfuran

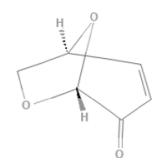

levoglucosenone

Fig. 1 Structures of the chemicals measured in this study. Structures were obtained from PubChem [24] 
the chromatogram and then accounting for any water by dividing by 1 plus the water content (if measured).

All samples except one had purities close to $100 \%$. The one exception was the levoglucosenone used for vapor pressure measurements, which only had a purity of $96.2 \mathrm{wt} \%$. Mass spectroscopy was used to investigate what impurities were present, and most of them were lower weight impurities such as 2-methylpentane, hexane, methylcyclopentane, cyclohexane and acetone. The levoglucosenone also contained one heavier impurity: 2-methoxyphenol. These impurities could be tolerated when measuring the vapor pressure because in the gas saturation method most of the light impurities are removed in the first run. This occurred with levoglucosenone in this study: the vapor pressure was much higher for the first run, and that point was removed. After the vapor pressure measurement, the purity of the levoglucosenone condensed at the outlet of the gas saturation cell was determined to be $98.5 \mathrm{wt} \%$. Later the levoglucosenone was distilled to get a higher purity for the density and refractive index measurements. This distilled sample had a purity of $98.7 \mathrm{wt} \%$.

\subsection{Density Measurements}

Densities were measured with a DMA HP density meter (Anton Paar). Most of the samples were measured at a range of pressures, and for these measurements a UNIK 5000 pressure sensor (GE) was used (range of 0 to $20 \mathrm{MPa}$, abs.). The sensor had been calibrated against a MC2-PE calibrator with an EXT600 external pressure module (Beamex). The MC2-PE calibrator had been calibrated by Beamex. The pressure data has a standard uncertainty of $3100 \mathrm{~Pa}$ (expanded uncertainty of $6300 \mathrm{~Pa}$ at the $95 \%$ level). For three of the samples measurements were only made at atmospheric pressure, and pressure data were taken from the Finnish Meteorological Institute (Tapiola observation station, Espoo, Finland) [25]. For the temperature, the manufacturer of the density meter only states that the accuracy is better than $0.1 \mathrm{~K}$.

The samples were degassed for about 30 min before measuring. This was done by placing the sample in a round-bottomed flask, which was then placed in an ultrasonic bath. Gasses were removed from the system using a vacuum pump.

Water and nitrogen were used to calibrate the density meter. Reference values for these compounds were taken from reference equations of state, $[26,27]$ and we used the implementations of these equations available in the CoolProp package for Python [28]. Alternatively, these equations of state are also implemented in the NIST thermophysical properties calculator, [29] and we verified the CoolProp implementation by manually comparing the results of the two programs at multiple temperatures and pressures. For optimizing the calibration equation parameters we used the differential evolution solver [30] implemented in the SciPy package [31] for Python. The root mean-squared error between the reference and calculated values was used as the objective function.

Between samples, the performance of the device was checked by measuring air and water. During the study the performance checks indicated that a recalibration was necessary, so for later samples, a second set of calibration parameters were used. 
For the first calibration the standard uncertainty was estimated to be $0.047 \mathrm{~kg} \cdot \mathrm{m}^{-3}$ (expanded uncertainty of $0.092 \mathrm{~kg} \cdot \mathrm{m}^{-3}$ at the $95 \%$ level). For the second calibration, the standard uncertainty was estimated to be $0.036 \mathrm{~kg} \cdot \mathrm{m}^{-3}$ (expanded uncertainty of $0.072 \mathrm{~kg} \cdot \mathrm{m}^{-3}$ at the $95 \%$ level). One major uncertainty component of density is the impurities in the sample. The impurities are sample specific and the effect is included in the uncertainty estimates given in the density results.

One sample, levoglucosenone, was measured at atmospheric pressure using a DMA 5000 M density meter (Anton Paar). The performance of the device was checked with water and air, and based on this the expanded uncertainty at the $95 \%$ level was estimated to be $0.05 \mathrm{~kg} \cdot \mathrm{m}^{-3}$.

Bio-compounds are often thermally unstable. The densities of decomposition products often deviate from that of the pure measured component. In some cases, this allows potential decomposition to be detected by just observing density changes during the measurement. When measuring furfural, decomposition was observed at $473 \mathrm{~K}$. Dihydrolevoglucosenone started to react at $423 \mathrm{~K}$. The density value was stable for about the first $20 \mathrm{~min}$ at this temperature, but then started to increase. Therefore, the few points from the beginning of the measurement at $423 \mathrm{~K}$ are included in the data file, but these points were not included during regression.

\subsection{Gas Saturation Measurements}

A gas saturation method was used to measure the vapor pressures of 6 of the biocompounds (2-methoxy-4-methylphenol, 2-sec-Butylphenol, 2,6-dimethoxyphenol, dihydrolevoglucosenone, levoglucosenone, and $\gamma$-valerolactone. About $10 \mathrm{ml}$ of each sample was placed in a glass vessel filled with spherical glass beads, and the vessel was put in a gas chromatography oven. The oven maintained a stable temperature (fluctuations were within $\pm 0.01 \mathrm{~K}$ ). A flow of nitrogen was introduced, and this gas became saturated with the vaporized compound. To maintain the flow rate of nitrogen, a flow controller (Alicat Scientific, Tucson, AZ, USA) was placed in the nitrogen inlet line. The nitrogen flow rate was measured with a bubble meter, both before and after each run (standard uncertainty of $0.039 \mathrm{ml} \cdot \mathrm{min}^{-1}$, expanded uncertainty of $0.088 \mathrm{ml} \cdot \mathrm{min}^{-1}$ at the $95 \%$ level). The vessel was left in the oven for a period of time (on the order of hours). Afterwards, it was removed and weighed to determine the mass lost. The vapor pressure was then calculated based on Eq. 1

$$
P=\frac{\frac{m}{W}}{\left(\frac{m}{W}+\frac{t V P_{\text {atm }}}{T_{\text {room }} R}\right)} \cdot\left(P_{\text {atm }}+\Delta P_{\text {loss }}\right)
$$

where $\mathrm{P}$ is the vapor pressure $(\mathrm{Pa}), \mathrm{m}$ is the mass of the test chemical that leaves the cell $(\mathrm{g}), \mathrm{W}$ is the molar mass of the test chemical $\left(\mathrm{g} \cdot \mathrm{mol}^{-1}\right), \mathrm{t}$ is the duration of the measurement ( $\mathrm{min}), \mathrm{V}$ is the volumetric flow rate of the carrier gas (in this case, nitrogen) in units of $\mathrm{L} \cdot \mathrm{min}^{-1}, \mathrm{P}_{\mathrm{atm}}$ is the atmospheric pressure at the place and time the experiment is carried out $(\mathrm{Pa}), \mathrm{T}_{\text {room }}$ is room temperature $(\mathrm{K}), \mathrm{R}$ is the ideal gas constant, and $\Delta \mathrm{P}_{\text {loss }}$ is the pressure drop over the gas saturation cell (assumed to be 
zero for our measurements). More details about the gas saturation method can be found from other references [32, 33].

Atmospheric pressure was taken from values measured by the Finnish Meteorological Institute (Tapiola observation station, Espoo, Finland) [25]. The cell and room temperatures were measured with calibrated Pt-100 temperature probes (Frontec) connected to a Systemteknik S2541 thermometer (Frontec). These probes had an expanded uncertainty of $0.04 \mathrm{~K}$ (using a coverage factor of 2 ).

The uncertainty of the vapor pressures were calculated using a Monte Carlo method (see ISO/IEC Guide 98-3) [34]. 8 different parameters were included that could potentially affect the vapor pressure value, including the purity of each compound. The uncertainty of those 8 parameters was used to specify a distribution for each, and values were then selected from those distributions to calculate a vapor pressure value. This was repeated 1 million times for each experimental data point. The standard uncertainty of each vapor pressure point was taken to be the standard deviation of the distribution from the Monte Carlo calculation. The calculated uncertainties can be found in the vapor pressure data file in the OSF project for this article (https://osf.io/u9amn/). The code we used for performing the uncertainty calculations can also be found in the same OSF project (https://osf.io/u9amn/).

\subsection{Refractive Index Measurements}

Refractive indexes were measured using a Dr. Kernchen Abbemat digital refractometer (Anton Paar, Graz, Austria), and this refractometer measures at a wavelength of $598.3 \mathrm{~nm}$. Based on measurements with water at $25^{\circ} \mathrm{C}$, the standard uncertainty of the refractometer was calculated to be 0.00034 (expanded uncertainty of 0.00078 at the $95 \%$ level). Reference data for water were obtained from Schiebener et al. [35].

\subsection{Modeling with PC-SAFT}

The PC-SAFT equation of state was used to model the properties of the bio-compounds [36]. Because all of the bio-compounds contain polar and/or associating functional groups, contributions from the dipole and associating terms were also included, as appropriate [37-42]. When including the dipole term from Gross and Vrabec, the equation is also called the PCP-SAFT equation of state [38].

De Villiers et al. [43] showed that it can be difficult to find the best fit for polar compounds using pure component data alone. Often there is a large range of parameter values that will give good results for pure component properties, but poor results for mixtures. De Villiers et al. suggested that this could be because it is difficult to disentangle the contribution from polar interactions from the part due to dispersion forces. They proposed including VLE data with a nonpolar component when fitting the pure component parameters for polar compounds. Because such data is not available for many of the bio-compounds studied in this article, we simply set the number of dipoles (a parameter in Gross and Vrabec's dipole term) equal to the actual number of polar functional groups in the molecule. This was the same strategy originally proposed by Gross and Vrabec [38]. The one exception was for furfural. A good fit 
could not be achieved unless the number of dipoles was also fitted against experimental data.

The dipole term also uses the dipole moment of the compound as a parameter. For most of the bio-compounds, the dipole moments were found in the literature [44-49]. Levoglucosenone was the only compound for which the dipole moment could not be found. Therefore, for use as a parameter in the PC-SAFT equation, the dipole moment was set to be equal to that of dihydrolevoglucosenone, since they are structurally similar compounds.

For some of the compounds in this article PC-SAFT parameters have already been presented in the literature (2-methylfuran, [50, 51]; 2-pentanone [37, 52]; cyclopentyl methyl ether [53]; furfural [37, 54]; tetrahydrofuran [37, 55] and $\gamma$-valerolactone [56]. We have refit parameters for these compounds because we had more data to include in the regression, including the new data measured in this article. Both literature data and data from this study were used when optimizing the PC-SAFT parameters. A file containing all the data used in optimization, including references, can be obtained from the OSF page (https://osf.io/u9amn/). It should be noted that in the data from Apaev et al. [57] for 2-pentanone there seems to have been a typo for the point at $376.63 \mathrm{~K}$ and 687 bar (probably should have been 799.5 instead of $899.5 \mathrm{~kg} \cdot \mathrm{m}^{-3}$ ), and we made this change in our literature data file. The total number of points used in the optimization and the number of literature data points is presented in Table 4. A total of 969 new measured data points were used in the regression.

Parameters were optimized by minimizing the root mean-squared errors of the vapor pressure and density added together. Optimization was performed using the differential evolution solver implemented in the Scipy package for Python [30, 31]. The resulting PC-SAFT parameters are given in Table 3. Our code for implementing the PC-SAFT equation of state can be found on GitHub: https://github.com/zmeri/ PC-SAFT.

\section{Results and Discussion}

\subsection{Compressed Liquid Density, Liquid Vapor Pressure and Refractive Index Measurement Results}

The results for the density measurement are presented in Tables 5, 6, 7, 8, 9, 10, $11,12,13$ and 14. The results for the vapor pressure measurement are presented in Tables 15, 16, 17, 18, 19 and 20. The measurements of the refractive index of the components can be found in Table 21 .

\subsection{Comparison with Literature Values}

A lot of literature data is available for tetrahydrofuran, and our experimental density values matched well with most of the literature values. For instance, at $293.15 \mathrm{~K}$ the value we measured was close to the mean of the literature data (see Table 22) and 


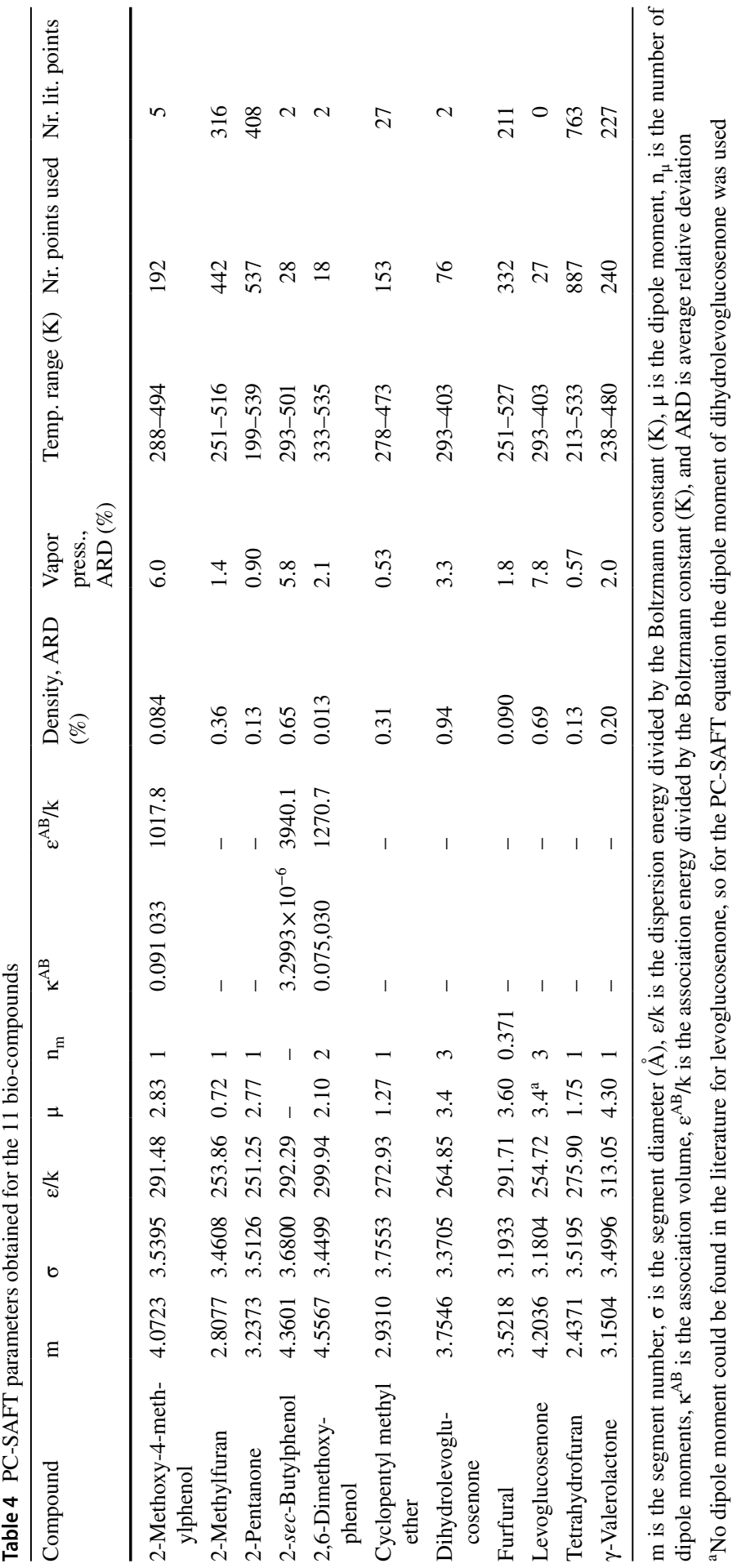


Table 5 Compressed liquid density of 2-methoxy-4-methylphenol (creosol)

\begin{tabular}{|c|c|c|c|c|c|}
\hline Pressure $(\mathrm{MPa})^{\mathrm{a}}$ & $\begin{array}{l}\text { Temperature } \\
(\mathrm{K})^{\mathrm{b}}\end{array}$ & $\begin{array}{l}\text { Density } \\
\left(\mathrm{kg} \mathrm{m}^{-3}\right)^{\mathrm{c}}\end{array}$ & Pressure $(\mathrm{MPa})^{\mathrm{a}}$ & $\begin{array}{l}\text { Temperature } \\
(\mathrm{K})^{\mathrm{b}}\end{array}$ & $\begin{array}{l}\text { Density } \\
\left(\mathrm{kg} \mathrm{m}^{-3}\right)^{\mathrm{c}}\end{array}$ \\
\hline 0.940 & 298.15 & 1092.38 & 1.025 & 433.16 & 962.89 \\
\hline 0.444 & 298.15 & 1092.14 & 0.527 & 433.15 & 962.10 \\
\hline 1.914 & 298.15 & 1092.96 & 0.144 & 433.15 & 961.62 \\
\hline 3.900 & 298.15 & 1094.10 & 1.509 & 433.15 & 963.22 \\
\hline 5.886 & 298.15 & 1095.18 & 2.005 & 433.14 & 963.80 \\
\hline 7.869 & 298.15 & 1096.19 & 2.501 & 433.15 & 964.34 \\
\hline 9.852 & 298.15 & 1097.24 & 2.996 & 433.15 & 964.90 \\
\hline 11.832 & 298.15 & 1098.23 & 3.493 & 433.15 & 965.46 \\
\hline 0.943 & 323.16 & 1069.03 & 3.989 & 433.15 & 966.01 \\
\hline 0.446 & 323.16 & 1068.70 & 4.487 & 433.15 & 966.52 \\
\hline 1.931 & 323.15 & 1069.66 & 4.982 & 433.15 & 967.09 \\
\hline 3.915 & 323.15 & 1070.89 & 5.478 & 433.14 & 967.66 \\
\hline 5.900 & 323.15 & 1072.11 & 5.974 & 433.15 & 968.16 \\
\hline 7.885 & 323.15 & 1073.27 & 1.035 & 433.15 & 962.66 \\
\hline 9.866 & 323.15 & 1074.46 & 1.019 & 413.14 & 982.66 \\
\hline 11.846 & 323.15 & 1075.60 & 0.536 & 413.14 & 982.20 \\
\hline 0.955 & 323.15 & 1069.04 & 0.135 & 413.15 & 981.75 \\
\hline 1.011 & 333.15 & 1059.62 & 1.510 & 413.15 & 983.16 \\
\hline 0.515 & 333.15 & 1059.29 & 2.004 & 413.14 & 983.64 \\
\hline 0.120 & 333.15 & 1059.04 & 2.499 & 413.14 & 984.16 \\
\hline 1.500 & 333.15 & 1059.96 & 2.997 & 413.15 & 984.65 \\
\hline 1.997 & 333.15 & 1060.29 & 3.492 & 413.15 & 985.12 \\
\hline 2.490 & 333.15 & 1060.62 & 3.990 & 413.15 & 985.63 \\
\hline 2.989 & 333.15 & 1060.95 & 4.485 & 413.15 & 986.10 \\
\hline 3.483 & 333.15 & 1061.28 & 4.984 & 413.14 & 986.63 \\
\hline 3.980 & 333.15 & 1061.61 & 5.480 & 413.14 & 987.09 \\
\hline 4.476 & 333.15 & 1061.89 & 5.976 & 413.14 & 987.61 \\
\hline 4.975 & 333.15 & 1062.22 & 1.030 & 413.15 & 982.64 \\
\hline 5.471 & 333.15 & 1062.55 & 1.028 & 298.15 & 1092.76 \\
\hline 5.967 & 333.15 & 1062.88 & 0.540 & 298.15 & 1092.48 \\
\hline 1.011 & 333.15 & 1059.67 & 0.145 & 298.15 & 1092.27 \\
\hline 1.030 & 293.15 & 1097.17 & 1.514 & 298.15 & 1093.06 \\
\hline 0.538 & 293.15 & 1096.88 & 2.011 & 298.15 & 1093.34 \\
\hline 0.135 & 293.15 & 1096.63 & 2.506 & 298.15 & 1093.63 \\
\hline 1.512 & 293.15 & 1097.51 & 3.003 & 298.15 & 1093.87 \\
\hline 2.009 & 293.15 & 1097.75 & 3.499 & 298.15 & 1094.15 \\
\hline 2.504 & 293.15 & 1098.04 & 3.996 & 298.15 & 1094.44 \\
\hline 3.003 & 293.15 & 1098.28 & 4.490 & 298.15 & 1094.72 \\
\hline 3.497 & 293.15 & 1098.56 & 4.988 & 298.15 & 1094.95 \\
\hline 3.994 & 293.15 & 1098.80 & 5.485 & 298.15 & 1095.23 \\
\hline 3.993 & 293.15 & 1098.75 & 5.981 & 298.15 & 1095.52 \\
\hline 4.490 & 293.15 & 1099.03 & 1.040 & 298.15 & 1092.77 \\
\hline
\end{tabular}


Table 5 (continued)

\begin{tabular}{|c|c|c|c|c|c|}
\hline Pressure $(\mathrm{MPa})^{\mathrm{a}}$ & $\begin{array}{l}\text { Temperature } \\
(\mathrm{K})^{\mathrm{b}}\end{array}$ & $\begin{array}{l}\text { Density } \\
\left(\mathrm{kg} \mathrm{m}^{-3}\right)^{\mathrm{c}}\end{array}$ & Pressure $(\mathrm{MPa})^{\mathrm{a}}$ & $\begin{array}{l}\text { Temperature } \\
(\mathrm{K})^{\mathrm{b}}\end{array}$ & $\begin{array}{l}\text { Density } \\
\left(\mathrm{kg} \mathrm{m}^{-3}\right)^{\mathrm{c}}\end{array}$ \\
\hline 4.988 & 293.15 & 1099.26 & 0.536 & 393.14 & 1001.91 \\
\hline 5.485 & 293.15 & 1099.54 & 0.144 & 393.14 & 1001.52 \\
\hline 5.982 & 293.15 & 1099.83 & 1.020 & 393.14 & 1002.33 \\
\hline 1.037 & 293.15 & 1097.12 & 1.510 & 393.14 & 1002.80 \\
\hline 1.038 & 313.16 & 1078.42 & 2.007 & 393.15 & 1003.21 \\
\hline 0.543 & 313.16 & 1078.14 & 2.503 & 393.15 & 1003.68 \\
\hline 0.134 & 313.15 & 1077.84 & 3.002 & 393.15 & 1004.10 \\
\hline 1.511 & 313.15 & 1078.72 & 3.496 & 393.15 & 1004.52 \\
\hline 1.511 & 313.15 & 1078.72 & 3.992 & 393.14 & 1005.00 \\
\hline 2.008 & 313.15 & 1079.01 & 4.488 & 393.14 & 1005.42 \\
\hline 2.504 & 313.15 & 1079.29 & 5.482 & 393.14 & 1006.31 \\
\hline 3.001 & 313.15 & 1079.63 & 4.987 & 393.14 & 1005.84 \\
\hline 3.497 & 313.15 & 1079.91 & 5.978 & 393.14 & 1006.73 \\
\hline 3.994 & 313.15 & 1080.19 & 1.032 & 393.15 & 1002.32 \\
\hline 4.488 & 313.15 & 1080.48 & 0.531 & 453.16 & 942.46 \\
\hline 4.989 & 313.15 & 1080.76 & 0.144 & 453.16 & 941.94 \\
\hline 5.483 & 313.15 & 1081.09 & 1.017 & 453.16 & 943.11 \\
\hline 5.980 & 313.15 & 1081.37 & 1.510 & 453.16 & 943.71 \\
\hline 1.040 & 313.15 & 1078.38 & 2.007 & 453.15 & 944.38 \\
\hline 1.022 & 353.16 & 1040.71 & 2.502 & 453.15 & 944.98 \\
\hline 0.540 & 353.16 & 1040.33 & 2.997 & 453.16 & 945.62 \\
\hline 0.135 & 353.15 & 1040.04 & 3.494 & 453.16 & 946.27 \\
\hline 1.510 & 353.15 & 1041.04 & 3.991 & 453.16 & 946.87 \\
\hline 2.006 & 353.15 & 1041.42 & 4.486 & 453.16 & 947.48 \\
\hline 2.503 & 353.15 & 1041.80 & 4.985 & 453.16 & 948.08 \\
\hline 3.001 & 353.15 & 1042.13 & 5.481 & 453.16 & 948.69 \\
\hline 3.497 & 353.15 & 1042.51 & 5.975 & 453.15 & 949.30 \\
\hline 3.991 & 353.15 & 1042.84 & 1.036 & 453.15 & 943.13 \\
\hline 4.489 & 353.15 & 1043.21 & 1.019 & 473.16 & 922.11 \\
\hline 4.987 & 353.15 & 1043.59 & 0.537 & 473.15 & 921.38 \\
\hline 5.483 & 353.15 & 1043.92 & 1.032 & 473.15 & 922.41 \\
\hline 5.980 & 353.15 & 1044.24 & 1.512 & 473.16 & 922.95 \\
\hline 1.034 & 353.15 & 1040.67 & 2.007 & 473.15 & 923.65 \\
\hline 1.017 & 373.16 & 1021.60 & 2.501 & 473.15 & 924.39 \\
\hline 0.533 & 373.16 & 1021.17 & 2.999 & 473.15 & 925.09 \\
\hline 0.133 & 373.15 & 1020.84 & 3.495 & 473.15 & 925.78 \\
\hline 1.510 & 373.15 & 1021.98 & 3.993 & 473.15 & 926.53 \\
\hline 2.007 & 373.15 & 1022.41 & 4.487 & 473.15 & 927.17 \\
\hline 2.503 & 373.15 & 1022.78 & 4.986 & 473.15 & 927.87 \\
\hline 2.999 & 373.15 & 1023.20 & 5.481 & 473.15 & 928.57 \\
\hline 3.496 & 373.16 & 1023.57 & 5.977 & 473.15 & 929.27 \\
\hline
\end{tabular}


Table 5 (continued)

\begin{tabular}{|c|c|c|c|c|c|}
\hline Pressure $(\mathrm{MPa})^{\mathrm{a}}$ & $\begin{array}{l}\text { Temperature } \\
(\mathrm{K})^{\mathrm{b}}\end{array}$ & $\begin{array}{l}\text { Density } \\
\left(\mathrm{kg} \mathrm{m}^{-3}\right)^{\mathrm{c}}\end{array}$ & Pressure $(\mathrm{MPa})^{\mathrm{a}}$ & $\begin{array}{l}\text { Temperature } \\
(\mathrm{K})^{\mathrm{b}}\end{array}$ & $\begin{array}{l}\text { Density } \\
\left(\mathrm{kg} \mathrm{m}^{-3}\right)^{\mathrm{c}}\end{array}$ \\
\hline 3.992 & 373.15 & 1023.96 & 0.538 & 473.15 & 921.53 \\
\hline 4.489 & 373.15 & 1024.34 & 1.030 & 473.15 & 922.46 \\
\hline 4.986 & 373.15 & 1024.76 & & & \\
\hline 5.481 & 373.15 & 1025.13 & & & \\
\hline 5.977 & 373.15 & 1025.55 & & & \\
\hline 1.031 & 373.15 & 1021.56 & & & \\
\hline
\end{tabular}

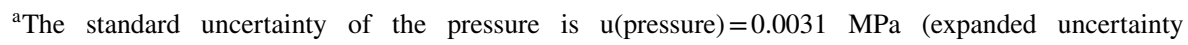
$\mathrm{u}($ pressure $)=0.0063 \mathrm{MPa}$ at the $95 \%$ level $)$

${ }^{\mathrm{b}}$ Standard uncertainty of temperature $\mathrm{u}($ temperature) $=0.1 \quad \mathrm{~K} \quad$ (expanded uncertainty $\mathrm{u}($ temperature $)=0.2 \mathrm{~K}$ at the $95 \%$ level)

${ }^{\mathrm{c}}$ Standard uncertainty of the density is $0.66 \mathrm{~kg} \cdot \mathrm{m}^{-3}$ (expanded uncertainty of $1.3 \mathrm{~kg} \cdot \mathrm{m}^{-3}$ at the $95 \%$ level)

was well within the standard deviation of the literature values $\left(0.33 \mathrm{~kg} \cdot \mathrm{m}^{-3}\right.$, with outliers removed).

We found seven literature sources that give compressed densities of tetrahydrofuran, and when comparing we found that the data from many of these literature sources seems to have relatively large errors [58-64]. Figure 2 compares the literature sources and values from this article, using the PC-SAFT fit for tetrahydrofuran as a reference. The points from Holzapfel et al. [58] and Sato et al. [59] aligned well with our data, as did much of the data from Govender et al. [60]. Data from Vasileva et al. [61] was in the same range as our data, but in general the deviations between the two data sets were larger than the measurement uncertainty of our data. The largest difference is $12 \mathrm{~kg} \cdot \mathrm{m}^{-3}$, and this occurs at $473.15 \mathrm{~K}$ and about 21 bar. At $293.15 \mathrm{~K}$ the value from Vasileva et al. is more than 7 standard deviations higher than the literature mean, and so it seems that there is a higher uncertainty in the data from Vasileva et al. The vapor pressures from Vasileva et al. also had some of the largest deviations from the PC-SAFT equation.

The data from the other three sources [62-64] was significantly lower than our values and those from other literature sources. Although these researchers mostly measured at much higher pressures than the other sources, values at atmospheric pressure show that these three data sets have significant errors. For instance, at $323.15 \mathrm{~K}$ and atmospheric pressure the value from Schornack and Eckert [62] is more than 35 standard deviations below the mean of the other literature values (ours was within a standard deviation). There may have been some problem with their experimental setup because their value for chlorobenzene at $323 \mathrm{~K}$ and atmospheric pressure is $10 \mathrm{~kg} \cdot \mathrm{m}^{-3}$ lower than the value from the DIPPR correlation [45], so there seems to be a consistent negative deviation. Zhang and Kiran [63] did not measure at atmospheric pressure, but extrapolating from their data down to atmospheric pressure using a linear pressure dependency shows a similar large negative deviation from most other literature sources. The uncertainty caused by the extrapolation is in this case a magnitude lower than the deviation. 
Table 6 Compressed liquid density of 2-Methylfuran

\begin{tabular}{|c|c|c|c|c|c|}
\hline Pressure $(\mathrm{MPa})^{\mathrm{a}}$ & $\begin{array}{l}\text { Temperature } \\
(\mathrm{K})^{\mathrm{b}}\end{array}$ & $\begin{array}{l}\text { Density (kg } \\
\left.\mathrm{m}^{-3}\right)^{\mathrm{c}}\end{array}$ & Pressure $(\mathrm{MPa})^{\mathrm{a}}$ & $\begin{array}{l}\text { Temperature } \\
(\mathrm{K})^{\mathrm{b}}\end{array}$ & $\begin{array}{l}\text { Density }(\mathrm{kg} \\
\left.\mathrm{m}^{-3}\right)^{\mathrm{c}}\end{array}$ \\
\hline 0.495 & 298.14 & 909.96 & 5.922 & 373.15 & 823.16 \\
\hline 0.095 & 298.14 & 909.46 & 6.912 & 373.15 & 824.89 \\
\hline 0.989 & 298.14 & 910.40 & 7.899 & 373.15 & 826.62 \\
\hline 1.974 & 298.14 & 911.38 & 8.885 & 373.15 & 828.29 \\
\hline 2.963 & 298.14 & 912.40 & 9.872 & 373.15 & 829.92 \\
\hline 3.952 & 298.14 & 913.42 & 10.856 & 373.15 & 831.50 \\
\hline 4.940 & 298.14 & 914.39 & 11.842 & 373.15 & 833.12 \\
\hline 5.929 & 298.14 & 915.36 & 1.004 & 398.15 & 777.14 \\
\hline 6.916 & 298.14 & 916.32 & 0.612 & 398.15 & 776.12 \\
\hline 7.904 & 298.14 & 917.28 & 1.973 & 398.16 & 779.62 \\
\hline 8.891 & 298.14 & 918.25 & 2.961 & 398.16 & 782.07 \\
\hline 9.877 & 298.14 & 919.16 & 3.946 & 398.16 & 784.46 \\
\hline 10.860 & 298.14 & 920.11 & 4.936 & 398.15 & 786.82 \\
\hline 11.847 & 298.14 & 921.02 & 5.924 & 398.15 & 789.07 \\
\hline 0.500 & 293.15 & 915.84 & 6.912 & 398.15 & 791.32 \\
\hline 0.088 & 293.14 & 915.46 & 7.898 & 398.15 & 793.47 \\
\hline 0.995 & 293.15 & 916.39 & 8.887 & 398.15 & 795.57 \\
\hline 1.972 & 293.15 & 917.36 & 9.874 & 398.16 & 797.61 \\
\hline 2.960 & 293.15 & 918.33 & 10.859 & 398.16 & 799.62 \\
\hline 3.949 & 293.15 & 919.30 & 11.843 & 398.16 & 801.52 \\
\hline 4.938 & 293.15 & 920.22 & 1.001 & 298.16 & 910.52 \\
\hline 5.926 & 293.15 & 921.19 & 0.989 & 423.15 & 736.05 \\
\hline 6.913 & 293.15 & 922.11 & 11.848 & 423.14 & 768.03 \\
\hline 7.899 & 293.15 & 923.02 & 11.845 & 423.14 & 768.38 \\
\hline 8.886 & 293.15 & 923.93 & 10.881 & 423.15 & 766.03 \\
\hline 9.874 & 293.15 & 924.85 & 11.843 & 423.15 & 768.37 \\
\hline 10.858 & 293.15 & 925.75 & 9.898 & 423.15 & 763.51 \\
\hline 11.843 & 293.15 & 926.61 & 8.918 & 423.15 & 760.94 \\
\hline 0.508 & 323.16 & 879.22 & 7.934 & 423.16 & 758.25 \\
\hline 0.092 & 323.15 & 878.68 & 6.948 & 423.16 & 755.49 \\
\hline 0.986 & 323.15 & 879.81 & 5.963 & 423.16 & 752.58 \\
\hline 11.847 & 323.15 & 892.53 & 4.967 & 423.15 & 749.60 \\
\hline 10.882 & 323.15 & 891.48 & 3.982 & 423.15 & 746.50 \\
\hline 9.902 & 323.15 & 890.43 & 2.990 & 423.15 & 743.21 \\
\hline 8.921 & 323.15 & 889.33 & 2.001 & 423.16 & 739.81 \\
\hline 7.935 & 323.15 & 888.22 & 1.005 & 423.16 & 736.18 \\
\hline 6.951 & 323.15 & 887.07 & 1.009 & 423.15 & 736.15 \\
\hline 5.959 & 323.15 & 885.91 & 11.843 & 423.15 & 768.40 \\
\hline 4.977 & 323.15 & 884.75 & 1.583 & 448.15 & 691.16 \\
\hline 3.984 & 323.15 & 883.55 & 11.844 & 448.14 & 733.04 \\
\hline 2.994 & 323.15 & 882.34 & 10.867 & 448.15 & 729.98 \\
\hline 1.998 & 323.15 & 881.12 & 9.895 & 448.15 & 726.76 \\
\hline
\end{tabular}


Table 6 (continued)

\begin{tabular}{|c|c|c|c|c|c|}
\hline Pressure $(\mathrm{MPa})^{\mathrm{a}}$ & $\begin{array}{l}\text { Temperature } \\
(\mathrm{K})^{\mathrm{b}}\end{array}$ & $\begin{array}{l}\text { Density (kg } \\
\left.\mathrm{m}^{-3}\right)^{\mathrm{c}}\end{array}$ & Pressure $(\mathrm{MPa})^{\mathrm{a}}$ & $\begin{array}{l}\text { Temperature } \\
(\mathrm{K})^{\mathrm{b}}\end{array}$ & $\begin{array}{l}\text { Density }(\mathrm{kg} \\
\left.\mathrm{m}^{-3}\right)^{\mathrm{c}}\end{array}$ \\
\hline 0.512 & 348.15 & 847.10 & 8.916 & 448.15 & 723.39 \\
\hline 11.845 & 348.15 & 863.31 & 7.931 & 448.15 & 719.79 \\
\hline 10.879 & 348.15 & 862.07 & 6.947 & 448.15 & 716.05 \\
\hline 9.896 & 348.15 & 860.78 & 5.956 & 448.14 & 712.07 \\
\hline 8.916 & 348.15 & 859.44 & 4.974 & 448.15 & 707.93 \\
\hline 7.933 & 348.15 & 858.09 & 3.982 & 448.15 & 703.52 \\
\hline 6.948 & 348.15 & 856.75 & 2.992 & 448.15 & 698.73 \\
\hline 5.961 & 348.15 & 855.31 & 2.000 & 448.14 & 693.56 \\
\hline 4.971 & 348.15 & 853.91 & 1.597 & 448.15 & 691.36 \\
\hline 3.976 & 348.15 & 852.42 & 2.277 & 473.15 & 637.03 \\
\hline 2.993 & 348.15 & 850.97 & 10.878 & 473.15 & 690.57 \\
\hline 1.995 & 348.15 & 849.42 & 11.843 & 473.15 & 694.63 \\
\hline 1.006 & 348.15 & 847.88 & 9.898 & 473.15 & 686.19 \\
\hline 0.212 & 348.15 & 846.65 & 8.910 & 473.14 & 681.52 \\
\hline 0.505 & 373.15 & 812.92 & 7.933 & 473.16 & 676.50 \\
\hline 0.406 & 373.15 & 812.69 & 6.947 & 473.15 & 671.08 \\
\hline 0.985 & 373.15 & 813.84 & 5.961 & 473.15 & 665.28 \\
\hline 1.971 & 373.15 & 815.77 & 4.969 & 473.15 & 658.86 \\
\hline 2.958 & 373.15 & 817.69 & 3.982 & 473.15 & 651.78 \\
\hline 3.946 & 373.15 & 819.52 & 2.990 & 473.15 & 643.66 \\
\hline 4.935 & 373.15 & 821.34 & 2.287 & 473.15 & 637.22 \\
\hline
\end{tabular}

${ }^{a}$ The standard uncertainty of the pressure is $\mathrm{u}$ (pressure) $=0.0031 \mathrm{MPa}$ (expanded uncertainty $\mathrm{u}($ pressure $)=0.0063 \mathrm{MPa}$ at the $95 \%$ level $)$

${ }^{\mathrm{b}}$ Standard uncertainty of temperature $\mathrm{u}$ (temperature) $=0.1 \quad \mathrm{~K} \quad$ (expanded uncertainty $\mathrm{u}($ temperature $)=0.2 \mathrm{~K}$ at the $95 \%$ level)

${ }^{\mathrm{c}}$ Standard uncertainty of the density is $0.11 \mathrm{~kg} \cdot \mathrm{m}^{-3}$ (expanded uncertainty of $0.21 \mathrm{~kg} \cdot \mathrm{m}^{-3}$ at the $95 \%$ level)

For instance, at $302.4 \mathrm{~K}$ we got a value of $848.2 \mathrm{~kg} \cdot \mathrm{m}^{-3}$ when extrapolating their data, which is about $28 \mathrm{~kg} \cdot \mathrm{m}^{-3}$ lower than the literature mean.

For the vapor pressure measurements gamma-valerolactone could be used as a reference compound for validating our gas saturation method. We found the literature data from 11 other sources, and we compared these different data sets in Fig. 3 [56, 65-74]. Most of the data fall within a few percentage points of each other, but there is one point from Havasi et al. [69] that is about $40 \%$ lower than the other data. It is so far out of line that it is below the y-axis limits in Fig. 3. In general, the data from Havasi et al. show an increasing negative deviation at lower temperatures, which could indicate a problem with the measurement procedure at those temperatures. The 3 points at the lowest temperatures from Havasi et al. were left out when fitting the PC-SAFT parameters. 
Table 7 Compressed liquid density of 2-pentanone

\begin{tabular}{|c|c|c|c|c|c|}
\hline Pressure $(\mathrm{MPa})^{\mathrm{a}}$ & $\begin{array}{l}\text { Temperature } \\
(\mathrm{K})^{\mathrm{b}}\end{array}$ & $\begin{array}{l}\text { Density } \\
\left(\mathrm{kg} \cdot \mathrm{m}^{-3}\right)^{\mathrm{c}}\end{array}$ & Pressure $(\mathrm{MPa})^{\mathrm{a}}$ & $\begin{array}{l}\text { Temperature } \\
(\mathrm{K})^{\mathrm{b}}\end{array}$ & $\begin{array}{l}\text { Density } \\
\left(\mathrm{kg} \cdot \mathrm{m}^{-3}\right)^{\mathrm{c}}\end{array}$ \\
\hline 0.495 & 298.14 & 801.70 & 7.900 & 373.15 & 736.41 \\
\hline 0.095 & 298.14 & 801.35 & 8.889 & 373.15 & 737.74 \\
\hline 0.987 & 298.15 & 802.14 & 9.874 & 373.15 & 739.02 \\
\hline 1.973 & 298.15 & 803.01 & 10.859 & 373.15 & 740.25 \\
\hline 2.962 & 298.15 & 803.87 & 11.845 & 373.15 & 741.48 \\
\hline 3.951 & 298.15 & 804.74 & 0.496 & 298.15 & 801.74 \\
\hline 4.939 & 298.15 & 805.56 & 0.492 & 398.16 & 697.96 \\
\hline 5.926 & 298.15 & 806.42 & 0.305 & 398.16 & 697.60 \\
\hline 6.917 & 298.15 & 807.23 & 11.847 & 398.16 & 717.17 \\
\hline 7.903 & 298.15 & 808.05 & 10.869 & 398.15 & 715.75 \\
\hline 8.890 & 298.15 & 808.86 & 9.905 & 398.15 & 714.30 \\
\hline 9.877 & 298.15 & 809.62 & 8.923 & 398.15 & 712.78 \\
\hline 10.862 & 298.15 & 810.43 & 7.941 & 398.15 & 711.22 \\
\hline 11.845 & 298.15 & 811.18 & 6.953 & 398.15 & 709.56 \\
\hline 0.497 & 293.15 & 806.56 & 5.965 & 398.15 & 707.89 \\
\hline 0.091 & 293.15 & 806.21 & 4.979 & 398.15 & 706.18 \\
\hline 0.986 & 293.15 & 806.95 & 3.987 & 398.15 & 704.43 \\
\hline 1.973 & 293.15 & 807.82 & 2.995 & 398.15 & 702.68 \\
\hline 2.962 & 293.15 & 808.64 & 2.004 & 398.15 & 700.88 \\
\hline 3.949 & 293.15 & 809.46 & 1.011 & 398.15 & 698.97 \\
\hline 4.940 & 293.15 & 810.28 & 0.999 & 423.15 & 669.40 \\
\hline 5.927 & 293.15 & 811.09 & 0.515 & 423.16 & 668.10 \\
\hline 6.915 & 293.15 & 811.86 & 11.848 & 423.15 & 692.13 \\
\hline 7.902 & 293.15 & 812.67 & 10.883 & 423.15 & 690.43 \\
\hline 8.889 & 293.15 & 813.44 & 9.903 & 423.14 & 688.59 \\
\hline 9.874 & 293.15 & 814.20 & 8.920 & 423.15 & 686.69 \\
\hline 10.860 & 293.15 & 814.96 & 7.938 & 423.15 & 684.75 \\
\hline 11.844 & 293.15 & 815.71 & 6.951 & 423.14 & 682.79 \\
\hline 0.509 & 323.16 & 777.26 & 5.966 & 423.14 & 680.75 \\
\hline 0.086 & 323.15 & 776.82 & 4.979 & 423.15 & 678.61 \\
\hline 0.987 & 323.15 & 777.75 & 3.986 & 423.15 & 676.39 \\
\hline 1.973 & 323.15 & 778.81 & 2.997 & 423.15 & 674.17 \\
\hline 2.961 & 323.15 & 779.82 & 2.003 & 423.14 & 671.87 \\
\hline 3.949 & 323.15 & 780.83 & 1.005 & 423.14 & 669.41 \\
\hline 4.940 & 323.15 & 781.79 & 0.987 & 448.16 & 636.76 \\
\hline 5.927 & 323.15 & 782.80 & 0.754 & 448.16 & 635.93 \\
\hline 6.914 & 323.15 & 783.75 & 11.847 & 448.15 & 665.96 \\
\hline 7.901 & 323.15 & 784.71 & 10.879 & 448.16 & 663.78 \\
\hline 8.889 & 323.15 & 785.66 & 9.903 & 448.16 & 661.56 \\
\hline 9.875 & 323.15 & 786.56 & 8.919 & 448.16 & 659.21 \\
\hline 10.859 & 323.15 & 787.47 & 7.937 & 448.16 & 656.81 \\
\hline 11.845 & 323.15 & 788.37 & 6.952 & 448.15 & 654.33 \\
\hline
\end{tabular}


Table 7 (continued)

\begin{tabular}{|c|c|c|c|c|c|}
\hline Pressure $(\mathrm{MPa})^{\mathrm{a}}$ & $\begin{array}{l}\text { Temperature } \\
(\mathrm{K})^{\mathrm{b}}\end{array}$ & $\begin{array}{l}\text { Density } \\
\left(\mathrm{kg} \cdot \mathrm{m}^{-3}\right)^{\mathrm{c}}\end{array}$ & Pressure $(\mathrm{MPa})^{\mathrm{a}}$ & $\begin{array}{l}\text { Temperature } \\
(\mathrm{K})^{\mathrm{b}}\end{array}$ & $\begin{array}{l}\text { Density } \\
\left(\mathrm{kg} \cdot \mathrm{m}^{-3}\right)^{\mathrm{c}}\end{array}$ \\
\hline 0.512 & 348.15 & 752.02 & 5.957 & 448.15 & 651.75 \\
\hline 0.086 & 348.15 & 751.49 & 4.976 & 448.16 & 649.05 \\
\hline 0.985 & 348.15 & 752.60 & 3.977 & 448.16 & 646.18 \\
\hline 1.972 & 348.15 & 753.85 & 2.993 & 448.16 & 643.21 \\
\hline 2.959 & 348.15 & 755.05 & 2.003 & 448.15 & 640.12 \\
\hline 3.948 & 348.15 & 756.24 & 1.010 & 448.15 & 636.87 \\
\hline 4.939 & 348.15 & 757.44 & 1.099 & 473.16 & 600.03 \\
\hline 5.925 & 348.15 & 758.59 & 11.849 & 473.15 & 638.34 \\
\hline 6.913 & 348.15 & 759.73 & 9.899 & 473.15 & 632.90 \\
\hline 7.902 & 348.15 & 760.87 & 7.939 & 473.15 & 626.90 \\
\hline 8.888 & 348.15 & 761.97 & 5.965 & 473.15 & 620.25 \\
\hline 9.876 & 348.15 & 763.06 & 3.986 & 473.14 & 612.91 \\
\hline 10.858 & 348.15 & 764.15 & 1.997 & 473.15 & 604.46 \\
\hline 11.845 & 348.15 & 765.20 & 1.104 & 473.15 & 600.13 \\
\hline 0.502 & 373.15 & 725.68 & 1.101 & 473.15 & 600.09 \\
\hline 0.114 & 373.15 & 725.04 & 2.961 & 473.15 & 608.54 \\
\hline 0.987 & 373.15 & 726.44 & 4.936 & 473.15 & 616.46 \\
\hline 1.972 & 373.15 & 727.92 & 6.913 & 473.14 & 623.50 \\
\hline 2.959 & 373.15 & 729.40 & 8.889 & 473.16 & 629.85 \\
\hline 3.948 & 373.15 & 730.84 & 10.859 & 473.15 & 635.67 \\
\hline 4.938 & 373.15 & 732.27 & 1.107 & 473.15 & 600.13 \\
\hline 5.925 & 373.15 & 733.70 & 0.496 & 298.15 & 801.73 \\
\hline 6.914 & 373.15 & 735.08 & & & \\
\hline
\end{tabular}

${ }^{\mathrm{a}}$ The standard uncertainty of the pressure is $\mathrm{u}$ (pressure) $=0.0031 \mathrm{MPa}$ (expanded uncertainty $\mathrm{u}($ pressure $)=0.0063 \mathrm{MPa}$ at the $95 \%$ level $)$

${ }^{\mathrm{b}}$ Standard uncertainty of temperature $\mathrm{u}$ (temperature) $=0.1 \quad \mathrm{~K} \quad$ (expanded uncertainty $\mathrm{u}$ (temperature $)=0.2 \mathrm{~K}$ at the $95 \%$ level $)$

${ }^{\mathrm{c}}$ Standard uncertainty of the density is $0.21 \mathrm{~kg} \cdot \mathrm{m}^{-3}$ (expanded uncertainty of $0.41 \mathrm{~kg} \cdot \mathrm{m}^{-3}$ at the $95 \%$ level)

Our data falls in line with the literature data, although it has a somewhat larger scatter than some of the literature data sets. In more recent experiments with the gas saturation equipment, we were able to improve the repeatability by making repeat measurements of the cell mass and taking the average of them, [75] so it seems that uncertainty in the sample weight was the largest contribution to the uncertainty for the gas saturation measurements presented here. We did not observe any systematic bias in our data when compared to the model or other data. 
Table 10 Compressed liquid density of cyclopentyl methyl ether

\begin{tabular}{|c|c|c|c|c|c|}
\hline Pressure $(\mathrm{MPa})^{\mathrm{a}}$ & $\begin{array}{l}\text { Temperature } \\
(\mathrm{K})^{\mathrm{b}}\end{array}$ & $\begin{array}{l}\text { Density } \\
\left(\mathrm{kg} \cdot \mathrm{m}^{-3}\right)^{\mathrm{c}}\end{array}$ & Pressure $(\mathrm{MPa})^{\mathrm{a}}$ & $\begin{array}{l}\text { Temperature } \\
(\mathrm{K})^{\mathrm{b}}\end{array}$ & $\begin{array}{l}\text { Density } \\
\left(\mathrm{kg} \cdot \mathrm{m}^{-3}\right)^{\mathrm{c}}\end{array}$ \\
\hline 0.505 & 298.14 & 858.52 & 5.926 & 373.15 & 790.28 \\
\hline 0.099 & 298.15 & 858.12 & 6.915 & 373.15 & 791.61 \\
\hline 0.990 & 298.15 & 858.91 & 7.900 & 373.15 & 792.89 \\
\hline 1.979 & 298.14 & 859.73 & 8.889 & 373.15 & 794.18 \\
\hline 2.965 & 298.14 & 860.56 & 9.876 & 373.15 & 795.41 \\
\hline 3.956 & 298.14 & 861.38 & 10.859 & 373.15 & 796.64 \\
\hline 4.943 & 298.14 & 862.20 & 11.845 & 373.15 & 797.83 \\
\hline 5.931 & 298.14 & 863.01 & 0.512 & 398.15 & 755.18 \\
\hline 6.920 & 298.14 & 863.78 & 0.310 & 398.15 & 754.82 \\
\hline 7.907 & 298.14 & 864.54 & 0.988 & 398.15 & 756.04 \\
\hline 8.894 & 298.14 & 865.35 & 1.973 & 398.15 & 757.80 \\
\hline 9.882 & 298.14 & 866.11 & 2.959 & 398.15 & 759.56 \\
\hline 10.867 & 298.14 & 866.87 & 3.950 & 398.15 & 761.22 \\
\hline 11.851 & 298.14 & 867.58 & 4.940 & 398.15 & 762.84 \\
\hline 0.514 & 293.14 & 863.31 & 5.927 & 398.15 & 764.46 \\
\hline 0.098 & 293.15 & 862.95 & 6.916 & 398.15 & 766.08 \\
\hline 0.988 & 293.15 & 863.69 & 7.902 & 398.15 & 767.59 \\
\hline 11.853 & 293.15 & 872.11 & 8.890 & 398.15 & 769.16 \\
\hline 10.886 & 293.15 & 871.40 & 9.876 & 398.15 & 770.62 \\
\hline 9.908 & 293.15 & 870.69 & 10.860 & 398.15 & 772.04 \\
\hline 8.927 & 293.15 & 869.98 & 11.845 & 398.15 & 773.46 \\
\hline 7.940 & 293.15 & 869.22 & 0.496 & 298.15 & 858.51 \\
\hline 6.955 & 293.15 & 868.45 & 1.002 & 423.15 & 726.95 \\
\hline 5.969 & 293.15 & 867.69 & 0.506 & 423.16 & 725.80 \\
\hline 4.977 & 293.15 & 866.92 & 11.851 & 423.16 & 748.37 \\
\hline 3.982 & 293.15 & 866.15 & 10.889 & 423.15 & 746.73 \\
\hline 3.000 & 293.15 & 865.33 & 9.909 & 423.15 & 744.99 \\
\hline 2.003 & 293.15 & 864.56 & 8.921 & 423.15 & 743.19 \\
\hline 0.513 & 323.16 & 834.09 & 7.943 & 423.16 & 741.38 \\
\hline 0.097 & 323.15 & 833.70 & 6.959 & 423.16 & 739.49 \\
\hline 0.988 & 323.15 & 834.58 & 5.964 & 423.16 & 737.54 \\
\hline 1.974 & 323.15 & 835.55 & 4.983 & 423.15 & 735.57 \\
\hline 2.962 & 323.15 & 836.56 & 3.987 & 423.15 & 733.54 \\
\hline 3.951 & 323.15 & 837.52 & 2.997 & 423.15 & 731.41 \\
\hline 4.942 & 323.15 & 838.43 & 2.002 & 423.16 & 729.21 \\
\hline 5.926 & 323.15 & 839.39 & 0.700 & 448.15 & 694.30 \\
\hline 6.916 & 323.15 & 840.30 & 11.852 & 448.15 & 722.29 \\
\hline 7.902 & 323.15 & 841.20 & 10.890 & 448.14 & 720.28 \\
\hline 8.889 & 323.15 & 842.11 & 9.906 & 448.14 & 718.20 \\
\hline 9.876 & 323.15 & 843.01 & 8.927 & 448.15 & 716.02 \\
\hline 10.861 & 323.15 & 843.92 & 7.944 & 448.15 & 713.76 \\
\hline 11.844 & 323.15 & 844.77 & 6.957 & 448.15 & 711.40 \\
\hline
\end{tabular}


Table 10 (continued)

\begin{tabular}{|c|c|c|c|c|c|}
\hline Pressure $(\mathrm{MPa})^{\mathrm{a}}$ & $\begin{array}{l}\text { Temperature } \\
(\mathrm{K})^{\mathrm{b}}\end{array}$ & $\begin{array}{l}\text { Density } \\
\left(\mathrm{kg} \cdot \mathrm{m}^{-3}\right)^{\mathrm{c}}\end{array}$ & Pressure $(\mathrm{MPa})^{\mathrm{a}}$ & $\begin{array}{l}\text { Temperature } \\
(\mathrm{K})^{\mathrm{b}}\end{array}$ & $\begin{array}{l}\text { Density } \\
\left(\mathrm{kg} \cdot \mathrm{m}^{-3}\right)^{\mathrm{c}}\end{array}$ \\
\hline 0.509 & 348.15 & 808.94 & 5.968 & 448.15 & 708.95 \\
\hline 0.092 & 348.15 & 808.41 & 4.982 & 448.15 & 706.45 \\
\hline 0.987 & 348.15 & 809.47 & 3.983 & 448.14 & 703.81 \\
\hline 11.852 & 348.15 & 821.55 & 2.997 & 448.15 & 701.12 \\
\hline 10.886 & 348.15 & 820.55 & 1.996 & 448.15 & 698.29 \\
\hline 9.905 & 348.15 & 819.56 & 1.010 & 448.15 & 695.32 \\
\hline 8.920 & 348.15 & 818.51 & 0.710 & 448.15 & 694.40 \\
\hline 7.942 & 348.15 & 817.42 & 11.851 & 473.14 & 694.93 \\
\hline 6.955 & 348.15 & 816.37 & 1.506 & 473.15 & 661.90 \\
\hline 5.963 & 348.15 & 815.27 & 1.011 & 473.15 & 659.73 \\
\hline 4.980 & 348.15 & 814.17 & 1.980 & 473.15 & 663.68 \\
\hline 3.986 & 348.15 & 813.02 & 2.965 & 473.15 & 667.54 \\
\hline 2.994 & 348.15 & 811.87 & 3.951 & 473.15 & 671.20 \\
\hline 2.004 & 348.15 & 810.73 & 4.940 & 473.15 & 674.68 \\
\hline 0.513 & 373.16 & 782.72 & 5.928 & 473.15 & 677.96 \\
\hline 0.107 & 373.16 & 782.14 & 6.916 & 473.15 & 681.07 \\
\hline 0.988 & 373.16 & 783.44 & 7.904 & 473.15 & 684.07 \\
\hline 1.971 & 373.16 & 784.83 & 8.889 & 473.15 & 686.99 \\
\hline 2.964 & 373.16 & 786.27 & 9.877 & 473.15 & 689.76 \\
\hline 3.948 & 373.15 & 787.60 & 10.861 & 473.15 & 692.39 \\
\hline 4.940 & 373.15 & 788.99 & 11.846 & 473.15 & 694.97 \\
\hline
\end{tabular}

${ }^{a}$ The standard uncertainty of the pressure is $\mathrm{u}$ (pressure) $=0.0031 \mathrm{MPa}$ (expanded uncertainty $\mathrm{u}$ (pressure $=0.0063 \mathrm{MPa}$ at the $95 \%$ level)

${ }^{\mathrm{b}}$ Standard uncertainty of temperature $\mathrm{u}($ temperature) $=0.1 \quad \mathrm{~K} \quad$ (expanded uncertainty $\mathrm{u}($ temperature $)=0.2 \mathrm{~K}$ at the $95 \%$ level)

${ }^{\mathrm{c}}$ Standard uncertainty of the density is $0.10 \mathrm{~kg} \mathrm{~m}^{-3}$ (expanded uncertainty of $0.20 \mathrm{~kg} \mathrm{~m}^{-3}$ at the $95 \%$ level)

Table 22 gives the density at $293 \mathrm{~K}$ for each of the 10 compounds measured in this study. Literature values are also given for comparison, where available. In general, our measured results match well with literature values. However, with 2-methoxy-4-methylphenol and 2-sec-butylphenol there are relatively large discrepancies between the experimental and literature values [75-101]. For both of these compounds only one literature value could be found, and they were from articles in 1952 and 1896 that used methods with higher uncertainties. The value for 2-pentanone may also seem to be out of line at first glance; however, when looking at the individual literature values one can see large variations between the different sources [82-100]. There are 5 sources that give a density close to ours (about $806.3 \mathrm{~kg} \cdot \mathrm{m}^{-3}$ ), but 6 of the 19 sources give a higher density of about $809 \mathrm{~kg} \cdot \mathrm{m}^{-3}$. So many of the values actually lie more than $1 \mathrm{~kg} \cdot \mathrm{m}^{-3}$ away from the mean. It is unclear why there is such a large scatter in the data for 2-pentanone, although all of the highest values 
Table 11 Compressed liquid density of dihydrolevoglucosenone

\begin{tabular}{|c|c|c|c|c|c|}
\hline Pressure $(\mathrm{MPa})^{\mathrm{a}}$ & $\begin{array}{l}\text { Temperature } \\
(\mathrm{K})^{\mathrm{b}}\end{array}$ & $\begin{array}{l}\text { Density } \\
\left(\mathrm{kg} \cdot \mathrm{m}^{-3}\right)^{\mathrm{c}}\end{array}$ & Pressure $(\mathrm{MPa})^{\mathrm{a}}$ & $\begin{array}{l}\text { Temperature } \\
(\mathrm{K})^{\mathrm{b}}\end{array}$ & $\begin{array}{l}\text { Density } \\
\left(\mathrm{kg} \cdot \mathrm{m}^{-3}\right)^{\mathrm{c}}\end{array}$ \\
\hline 0.090 & 293.15 & 1250.75 & 15.756 & 348.15 & 1207.18 \\
\hline 3.943 & 293.15 & 1252.88 & 0.088 & 348.15 & 1196.86 \\
\hline 9.868 & 293.15 & 1256.00 & 1.953 & 348.15 & 1198.15 \\
\hline 13.804 & 293.15 & 1257.92 & 3.930 & 348.15 & 1199.47 \\
\hline 15.764 & 293.15 & 1258.89 & 5.907 & 348.15 & 1200.83 \\
\hline 5.919 & 293.15 & 1253.91 & 7.882 & 348.15 & 1202.14 \\
\hline 7.894 & 293.15 & 1254.94 & 5.906 & 373.15 & 1176.75 \\
\hline 11.837 & 293.15 & 1256.99 & 1.978 & 373.15 & 1173.80 \\
\hline 1.967 & 293.15 & 1251.77 & 3.928 & 373.15 & 1175.25 \\
\hline 0.093 & 298.15 & 1245.77 & 7.881 & 373.15 & 1178.24 \\
\hline 0.984 & 298.15 & 1246.32 & 0.086 & 373.15 & 1172.28 \\
\hline 9.872 & 298.15 & 1251.16 & 9.856 & 373.15 & 1179.67 \\
\hline 13.808 & 298.15 & 1253.14 & 11.824 & 373.15 & 1181.11 \\
\hline 15.768 & 298.15 & 1254.11 & 13.791 & 373.15 & 1182.49 \\
\hline 1.947 & 298.15 & 1246.95 & 15.754 & 373.15 & 1183.87 \\
\hline 1.948 & 298.15 & 1247.00 & 1.952 & 373.16 & 1173.74 \\
\hline 1.969 & 298.15 & 1246.85 & 5.902 & 398.15 & 1152.51 \\
\hline 3.946 & 298.15 & 1247.95 & 7.879 & 398.15 & 1154.19 \\
\hline 5.923 & 298.15 & 1249.03 & 9.852 & 398.15 & 1155.82 \\
\hline 7.897 & 298.15 & 1250.10 & 11.823 & 398.15 & 1157.44 \\
\hline 11.843 & 298.15 & 1252.16 & 13.789 & 398.15 & 1159.02 \\
\hline 0.084 & 323.15 & 1221.30 & 15.749 & 398.15 & 1160.55 \\
\hline 1.957 & 323.15 & 1222.51 & 1.976 & 398.15 & 1149.35 \\
\hline 3.933 & 323.15 & 1223.70 & 3.926 & 398.15 & 1150.87 \\
\hline 5.912 & 323.15 & 1224.92 & 1.949 & 398.16 & 1149.14 \\
\hline 7.886 & 323.15 & 1226.08 & 0.082 & 398.16 & 1147.60 \\
\hline 9.860 & 323.15 & 1227.24 & 1.958 & 423.15 & 1124.40 \\
\hline 11.830 & 323.15 & 1228.38 & 0.073 & 423.14 & 1122.74 \\
\hline 13.795 & 323.15 & 1229.51 & 0.091 & 423.15 & 1122.77 \\
\hline 15.760 & 323.15 & 1230.58 & 1.950 & 423.16 & 1124.38 \\
\hline 9.856 & 348.15 & 1203.43 & 15.756 & 423.16 & 1137.14 \\
\hline 11.827 & 348.15 & 1204.67 & 9.888 & 423.14 & 1131.94 \\
\hline 13.792 & 348.15 & 1205.96 & 1.971 & 423.14 & 1124.61 \\
\hline
\end{tabular}

${ }^{\mathrm{a}}$ The standard uncertainty of the pressure is $\mathrm{u}$ (pressure) $=0.0031 \mathrm{MPa}$ (expanded uncertainty $\mathrm{u}($ pressure $)=0.0063 \mathrm{MPa}$ at the $95 \%$ level $)$

${ }^{\mathrm{b}}$ Standard uncertainty of temperature $\mathrm{u}($ temperature) $=0.1 \quad \mathrm{~K} \quad$ (expanded uncertainty $\mathrm{u}($ temperature $)=0.2 \mathrm{~K}$ at the $95 \%$ level $)$

${ }^{\mathrm{c}}$ Standard uncertainty of the density is $0.25 \mathrm{~kg} \cdot \mathrm{m}^{-3}$ (expanded uncertainty of $0.51 \mathrm{~kg} \cdot \mathrm{m}^{-3}$ at the $95 \%$ level) 
Table 12 Compressed liquid density of furfural

\begin{tabular}{|c|c|c|c|c|c|}
\hline Pressure $(\mathrm{MPa})^{\mathrm{a}}$ & $\begin{array}{l}\text { Temperature } \\
(\mathrm{K})^{\mathrm{b}}\end{array}$ & $\begin{array}{l}\text { Density } \\
\left(\mathrm{kg} \cdot \mathrm{m}^{-3}\right)^{\mathrm{c}}\end{array}$ & Pressure $(\mathrm{MPa})^{\mathrm{a}}$ & $\begin{array}{l}\text { Temperature } \\
(\mathrm{K})^{\mathrm{b}}\end{array}$ & $\begin{array}{l}\text { Density } \\
\left(\mathrm{kg} \cdot \mathrm{m}^{-3}\right)^{\mathrm{c}}\end{array}$ \\
\hline 0.497 & 293.15 & 1160.04 & 1.974 & 373.15 & 1074.66 \\
\hline 0.092 & 293.15 & 1159.79 & 2.959 & 373.15 & 1075.61 \\
\hline 0.987 & 293.15 & 1160.38 & 3.949 & 373.15 & 1076.51 \\
\hline 1.973 & 293.15 & 1161.01 & 4.940 & 373.15 & 1077.41 \\
\hline 2.961 & 293.15 & 1161.64 & 5.929 & 373.15 & 1078.35 \\
\hline 3.950 & 293.15 & 1162.22 & 6.916 & 373.15 & 1079.25 \\
\hline 4.940 & 293.15 & 1162.84 & 7.903 & 373.15 & 1080.14 \\
\hline 5.930 & 293.15 & 1163.46 & 8.890 & 373.15 & 1080.99 \\
\hline 6.920 & 293.15 & 1164.07 & 9.878 & 373.15 & 1081.88 \\
\hline 7.905 & 293.15 & 1164.64 & 10.863 & 373.15 & 1082.73 \\
\hline 8.894 & 293.15 & 1165.24 & 11.849 & 373.15 & 1083.57 \\
\hline 9.881 & 293.15 & 1165.85 & 0.510 & 373.15 & 1073.27 \\
\hline 10.865 & 293.15 & 1166.40 & 0.495 & 398.16 & 1044.93 \\
\hline 11.851 & 293.15 & 1167.00 & 0.092 & 398.16 & 1044.54 \\
\hline 0.495 & 298.14 & 1154.82 & 0.987 & 398.15 & 1045.45 \\
\hline 0.093 & 298.14 & 1154.52 & 11.856 & 398.15 & 1056.72 \\
\hline 0.988 & 298.15 & 1155.06 & 10.874 & 398.15 & 1055.78 \\
\hline 1.973 & 298.15 & 1155.69 & 9.894 & 398.15 & 1054.84 \\
\hline 2.962 & 298.15 & 1156.32 & 8.912 & 398.15 & 1053.80 \\
\hline 3.952 & 298.15 & 1156.99 & 7.928 & 398.15 & 1052.78 \\
\hline 4.941 & 298.15 & 1157.61 & 6.942 & 398.15 & 1051.79 \\
\hline 5.929 & 298.15 & 1158.23 & 5.954 & 398.15 & 1050.80 \\
\hline 6.917 & 298.15 & 1158.84 & 4.966 & 398.15 & 1049.76 \\
\hline 7.904 & 298.15 & 1159.45 & 3.974 & 398.15 & 1048.73 \\
\hline 8.893 & 298.15 & 1160.06 & 2.983 & 398.15 & 1047.64 \\
\hline 9.878 & 298.15 & 1160.67 & 1.990 & 398.15 & 1046.59 \\
\hline 10.864 & 298.15 & 1161.27 & 0.518 & 398.15 & 1044.99 \\
\hline 11.849 & 298.15 & 1161.82 & 0.494 & 423.16 & 1015.70 \\
\hline 0.514 & 298.15 & 1154.81 & 0.108 & 423.14 & 1015.20 \\
\hline 0.499 & 298.15 & 1154.81 & 0.986 & 423.14 & 1016.34 \\
\hline 0.496 & 323.15 & 1128.08 & 11.854 & 423.14 & 1029.37 \\
\hline 0.089 & 323.15 & 1127.78 & 9.892 & 423.15 & 1027.14 \\
\hline 0.986 & 323.15 & 1128.47 & 7.925 & 423.15 & 1024.83 \\
\hline 1.974 & 323.15 & 1129.14 & 5.953 & 423.14 & 1022.50 \\
\hline 2.962 & 323.15 & 1129.86 & 3.975 & 423.14 & 1020.10 \\
\hline 3.951 & 323.15 & 1130.53 & 0.515 & 423.15 & 1015.76 \\
\hline 4.940 & 323.15 & 1131.24 & 1.976 & 423.15 & 1017.54 \\
\hline 5.929 & 323.15 & 1131.96 & 2.964 & 423.15 & 1018.77 \\
\hline 6.917 & 323.15 & 1132.62 & 4.941 & 423.14 & 1021.23 \\
\hline 7.903 & 323.15 & 1133.32 & 6.917 & 423.14 & 1023.63 \\
\hline 8.890 & 323.15 & 1133.98 & 8.891 & 423.15 & 1025.96 \\
\hline 9.878 & 323.15 & 1134.68 & 10.863 & 423.15 & 1028.22 \\
\hline
\end{tabular}


Table 12 (continued)

\begin{tabular}{lllllr}
\hline Pressure $(\mathrm{MPa})^{\mathrm{a}}$ & $\begin{array}{l}\text { Temperature } \\
(\mathrm{K})^{\mathrm{b}}\end{array}$ & $\begin{array}{l}\text { Density } \\
\left(\mathrm{kg} \cdot \mathrm{m}^{-3}\right)^{\mathrm{c}}\end{array}$ & Pressure $(\mathrm{MPa})^{\mathrm{a}}$ & $\begin{array}{l}\text { Temperature } \\
(\mathrm{K})^{\mathrm{b}}\end{array}$ & $\begin{array}{l}\text { Density } \\
\left(\mathrm{kg} \cdot \mathrm{m}^{-3}\right)^{\mathrm{c}}\end{array}$ \\
\hline 10.862 & 323.15 & 1135.33 & 0.514 & 423.16 & 1015.74 \\
11.850 & 323.15 & 1135.98 & 0.491 & 448.15 & 985.23 \\
0.495 & 348.16 & 1100.92 & 0.298 & 448.14 & 984.99 \\
0.087 & 348.15 & 1100.58 & 11.852 & 448.15 & 1001.25 \\
0.988 & 348.15 & 1101.31 & 9.891 & 448.15 & 998.65 \\
1.971 & 348.15 & 1102.12 & 7.924 & 448.15 & 996.02 \\
2.961 & 348.15 & 1102.93 & 5.951 & 448.15 & 993.24 \\
3.950 & 348.15 & 1103.74 & 3.970 & 448.15 & 990.43 \\
4.940 & 348.15 & 1104.55 & 1.987 & 448.14 & 987.54 \\
5.928 & 348.15 & 1105.31 & 0.514 & 448.15 & 985.33 \\
6.917 & 348.15 & 1106.11 & 0.513 & 448.15 & 985.28 \\
7.904 & 348.15 & 1106.91 & 0.988 & 448.14 & 986.04 \\
8.891 & 348.15 & 1107.66 & 2.962 & 448.15 & 988.93 \\
9.879 & 348.15 & 1108.41 & 4.940 & 448.15 & 991.79 \\
10.863 & 348.15 & 1109.16 & 6.917 & 448.15 & 994.56 \\
11.849 & 348.15 & 1109.91 & 8.892 & 448.15 & 997.29 \\
0.500 & 373.16 & 1073.32 & 10.863 & 448.14 & 999.94 \\
0.091 & 373.15 & 1072.93 & 0.509 & 448.15 & 985.37 \\
0.988 & 373.16 & 1073.70 & & & \\
\hline
\end{tabular}

${ }^{a}$ The standard uncertainty of the pressure is $\mathrm{u}$ (pressure) $=0.0031 \mathrm{MPa}$ (expanded uncertainty $\mathrm{u}($ pressure $)=0.0063 \mathrm{MPa}$ at the $95 \%$ level $)$

${ }^{\mathrm{b}}$ Standard uncertainty of temperature $\mathrm{u}$ (temperature) $=0.1 \quad \mathrm{~K} \quad$ (expanded uncertainty $\mathrm{u}($ temperature $)=0.2 \mathrm{~K}$ at the $95 \%$ level)

${ }^{\mathrm{c}}$ Standard uncertainty of the density is $0.24 \mathrm{~kg} \cdot \mathrm{m}^{-3}$ (expanded uncertainty of $0.47 \mathrm{~kg} \cdot \mathrm{m}^{-3}$ at the $95 \%$ level)

were measured more than 50 years ago, and the more recent articles report a lower density.

Using the PC-SAFT equation, we could calculate the normal boiling point (at $101,325 \mathrm{~Pa}$ ) and enthalpy of vaporization of each compound. These values are given in Table 23. For dihydrolevoglucosenone and levoglucosenone the PC-SAFT equation had to be extrapolated about $100 \mathrm{~K}$ above the available vapor pressure data to reach the boiling point, so the boiling points for these compounds contain larger uncertainty. For dihydrolevoglucosenone Sherwood et al. [3] measured an approximate value of $476 \mathrm{~K}$ for the boiling point using a TGA, which is about $20 \mathrm{~K}$ lower than the value we calculated. We observed that dihydrolevoglucosenone decomposed even at $423 \mathrm{~K}$ (see Sect. 2.2), and so it is possible that decomposition occurred in the TGA measurement, leading to an estimated value lower than the actual boiling point.

We can also briefly examine the relationships between molecular structure and properties. For instance we can see that hydrogenating levoglucosenone to 
Table 14 Compressed liquid density of tetrahydrofuran

\begin{tabular}{|c|c|c|c|c|c|}
\hline Pressure $(\mathrm{MPa})^{\mathrm{a}}$ & Temperature $(\mathrm{K})^{\mathrm{b}}$ & Density $\left(\mathrm{kg} \cdot \mathrm{m}^{-3}\right)^{\mathrm{c}}$ & Pressure $(\mathrm{MPa})^{\mathrm{a}}$ & Temperature $(\mathrm{K})^{\mathrm{b}}$ & $\begin{array}{l}\text { Density } \\
\left(\mathrm{kg} \cdot \mathrm{m}^{-3}\right)^{\mathrm{c}}\end{array}$ \\
\hline 0.402 & 298.15 & 882.38 & 9.901 & 348.15 & 837.19 \\
\hline 0.099 & 298.15 & 882.02 & 8.920 & 348.15 & 836.10 \\
\hline 0.992 & 298.15 & 882.81 & 7.933 & 348.15 & 834.96 \\
\hline 11.854 & 298.15 & 891.84 & 6.953 & 348.15 & 833.81 \\
\hline 10.874 & 298.15 & 891.09 & 5.962 & 348.15 & 832.61 \\
\hline 9.893 & 298.15 & 890.29 & 4.979 & 348.15 & 831.47 \\
\hline 8.909 & 298.15 & 889.53 & 3.987 & 348.15 & 830.22 \\
\hline 7.925 & 298.15 & 888.73 & 2.997 & 348.15 & 829.02 \\
\hline 6.941 & 298.15 & 887.92 & 2.006 & 348.15 & 827.77 \\
\hline 5.954 & 298.15 & 887.11 & 0.215 & 348.15 & 825.49 \\
\hline 4.965 & 298.15 & 886.24 & 0.988 & 373.15 & 796.10 \\
\hline 3.977 & 298.15 & 885.42 & 1.974 & 373.16 & 797.63 \\
\hline 2.986 & 298.15 & 884.55 & 2.960 & 373.15 & 799.18 \\
\hline 1.994 & 298.15 & 883.68 & 3.948 & 373.15 & 800.67 \\
\hline 0.513 & 298.15 & 882.42 & 4.937 & 373.15 & 802.14 \\
\hline 0.502 & 293.15 & 887.82 & 5.925 & 373.15 & 803.62 \\
\hline 0.111 & 293.15 & 887.47 & 6.910 & 373.15 & 805.06 \\
\hline 11.854 & 293.15 & 896.95 & 7.898 & 373.15 & 806.44 \\
\hline 0.494 & 293.15 & 887.77 & 8.886 & 373.15 & 807.82 \\
\hline 11.854 & 293.15 & 896.96 & 9.874 & 373.15 & 809.20 \\
\hline 10.892 & 293.15 & 896.25 & 10.857 & 373.15 & 810.53 \\
\hline 9.910 & 293.15 & 895.50 & 11.842 & 373.15 & 811.81 \\
\hline 1.013 & 293.14 & 888.32 & 0.512 & 373.15 & 795.44 \\
\hline 1.986 & 293.15 & 889.09 & 0.313 & 373.15 & 795.02 \\
\hline 2.968 & 293.15 & 889.92 & 0.500 & 398.16 & 762.57 \\
\hline 3.953 & 293.15 & 890.74 & 1.974 & 398.15 & 765.55 \\
\hline 4.940 & 293.15 & 891.56 & 11.848 & 398.15 & 783.16 \\
\hline 5.928 & 293.15 & 892.37 & 10.867 & 398.15 & 781.60 \\
\hline 6.917 & 293.15 & 893.14 & 9.898 & 398.15 & 780.08 \\
\hline 7.904 & 293.15 & 893.95 & 8.916 & 398.16 & 778.41 \\
\hline 8.890 & 293.15 & 894.70 & 7.933 & 398.16 & 776.74 \\
\hline 0.512 & 323.15 & 854.73 & 6.943 & 398.16 & 774.98 \\
\hline 0.103 & 323.15 & 854.29 & 5.965 & 398.15 & 773.22 \\
\hline 0.986 & 323.15 & 855.22 & 4.975 & 398.15 & 771.41 \\
\hline 11.852 & 323.15 & 865.93 & 3.985 & 398.15 & 769.55 \\
\hline 10.885 & 323.15 & 865.03 & 2.995 & 398.15 & 767.65 \\
\hline 9.905 & 323.15 & 864.13 & 1.007 & 398.16 & 763.67 \\
\hline 8.924 & 323.15 & 863.18 & 0.899 & 423.15 & 727.70 \\
\hline 7.940 & 323.15 & 862.23 & 0.990 & 423.16 & 727.96 \\
\hline 6.949 & 323.15 & 861.28 & 11.847 & 423.16 & 753.18 \\
\hline 5.970 & 323.15 & 860.32 & 2.002 & 423.15 & 730.83 \\
\hline 4.978 & 323.15 & 859.32 & 2.969 & 423.16 & 733.24 \\
\hline 3.987 & 323.15 & 858.31 & 3.954 & 423.16 & 735.70 \\
\hline
\end{tabular}


Table 14 (continued)

\begin{tabular}{|c|c|c|c|c|c|}
\hline Pressure $(\mathrm{MPa})^{\mathrm{a}}$ & Temperature $(\mathrm{K})^{\mathrm{b}}$ & Density $\left(\mathrm{kg} \cdot \mathrm{m}^{-3}\right)^{\mathrm{c}}$ & Pressure $(\mathrm{MPa})^{\mathrm{a}}$ & Temperature $(\mathrm{K})^{\mathrm{b}}$ & $\begin{array}{l}\text { Density } \\
\left(\mathrm{kg} \cdot \mathrm{m}^{-3}\right)^{\mathrm{c}}\end{array}$ \\
\hline 3.001 & 323.15 & 857.30 & 4.940 & 423.16 & 738.08 \\
\hline 2.002 & 323.15 & 856.28 & 5.929 & 423.15 & 740.47 \\
\hline 1.998 & 443.14 & 699.53 & 6.914 & 423.15 & 742.75 \\
\hline 2.972 & 443.14 & 702.90 & 7.901 & 423.15 & 744.94 \\
\hline 11.849 & 443.15 & 727.80 & 8.886 & 423.16 & 747.10 \\
\hline 3.986 & 443.15 & 706.29 & 9.879 & 423.16 & 749.19 \\
\hline 4.957 & 443.15 & 709.28 & 10.857 & 423.15 & 751.20 \\
\hline 5.940 & 443.15 & 712.17 & 2.094 & 473.16 & 644.15 \\
\hline 6.925 & 443.15 & 715.01 & 11.846 & 473.15 & 686.73 \\
\hline 7.909 & 443.14 & 717.78 & 10.872 & 473.15 & 683.48 \\
\hline 8.895 & 443.15 & 720.41 & 9.898 & 473.15 & 680.08 \\
\hline 9.880 & 443.15 & 722.97 & 8.910 & 473.15 & 676.45 \\
\hline 10.865 & 443.15 & 725.44 & 7.932 & 473.15 & 672.68 \\
\hline 1.393 & 443.15 & 697.40 & 6.948 & 473.15 & 668.67 \\
\hline 1.017 & 298.15 & 882.85 & 5.961 & 473.15 & 664.38 \\
\hline 0.509 & 348.15 & 825.84 & 4.975 & 473.15 & 659.81 \\
\hline 0.992 & 348.15 & 826.47 & 3.982 & 473.15 & 654.91 \\
\hline 11.846 & 348.15 & 839.27 & 2.985 & 473.15 & 649.50 \\
\hline 10.882 & 348.15 & 838.28 & 2.494 & 473.15 & 646.62 \\
\hline
\end{tabular}

${ }^{\mathrm{a}}$ The standard uncertainty of the pressure is $\mathrm{u}$ (pressure) $=0.0031 \mathrm{MPa}$ (expanded uncertainty $\mathrm{u}($ pressure $)=0.0063 \mathrm{MPa}$ at the $95 \%$ level)

${ }^{\mathrm{b}}$ Standard uncertainty of temperature $\mathrm{u}$ (temperature) $=0.1 \quad \mathrm{~K} \quad$ (expanded uncertainty $\mathrm{u}($ temperature $)=0.2 \mathrm{~K}$ at the $95 \%$ level)

${ }^{\mathrm{c}}$ Standard uncertainty of the density is $0.1 \mathrm{~kg} \cdot \mathrm{m}^{-3}$ (expanded uncertainty of $0.2 \mathrm{~kg} \cdot \mathrm{m}^{-3}$ at the $95 \%$ level)

Table 15 Vapor pressure of liquid 2-methoxy-4methylphenol

\begin{tabular}{lll}
\hline Temperature $\mathrm{K}^{\mathrm{a}}$ & $\begin{array}{l}\text { Vapor pressure } \\
\text { Liquid Pa }\end{array}$ & $\begin{array}{l}\text { Expanded uncertainty of } \\
\text { vapor pressure at 95 \% } \\
\text { level Pa }\end{array}$ \\
\hline 298.22 & 9.3 & 1.0 \\
303.24 & 16.1 & 1.4 \\
313.25 & 31.7 & 3.0 \\
323.24 & 81.8 & 6.4 \\
323.25 & 82.1 & 6.2 \\
333.27 & 143.0 & 6.3 \\
343.22 & 274.5 & 9.2 \\
353.27 & 476 & 20 \\
363.22 & 807 & 42 \\
373.21 & 1533 & 58 \\
383.22 & 2280 & 119 \\
393.16 & 3520 & 122 \\
403.20 & 5320 & 141 \\
\hline
\end{tabular}

${ }^{\mathrm{a}}$ Standard uncertainty of the temperature $\mathrm{u}(\mathrm{T})=0.04 \mathrm{~K}$ 
Table 16 Vapor pressure of liquid 2-sec-butylphenol

Table 17 Vapor pressure of liquid 2,6-dimethoxyphenol (syringol)

\begin{tabular}{lll}
\hline Temperature $\mathrm{K}^{\mathrm{a}}$ & $\begin{array}{l}\text { Vapor pressure } \\
\text { liquid }(\mathrm{Pa})\end{array}$ & $\begin{array}{l}\text { Expanded uncertainty of } \\
\text { vapor pressure at } 95 \% \text { level } \\
(\mathrm{Pa})\end{array}$ \\
\hline 298.21 & 4.74 & 0.74 \\
303.24 & 8.5 & 1.3 \\
313.25 & 18.4 & 2.5 \\
323.25 & 49.4 & 5.5 \\
333.29 & 94.2 & 11 \\
343.23 & 231 & 24 \\
343.24 & 226.6 & 6.9 \\
353.28 & 449 & 23 \\
363.23 & 663 & 21 \\
363.26 & 659 & 35 \\
373.25 & 1097 & 60 \\
383.31 & 1868 & 96 \\
393.25 & 2940 & 126 \\
403.25 & 4570 & 139 \\
\hline
\end{tabular}

${ }^{\mathrm{a}}$ Standard uncertainty of the temperature $\mathrm{u}(\mathrm{T})=0.04 \mathrm{~K}$

\begin{tabular}{lll}
\hline Temperature $\mathrm{K}^{\mathrm{a}}$ & $\begin{array}{l}\text { Vapor pressure } \\
\text { liquid }(\mathrm{Pa})\end{array}$ & $\begin{array}{l}\text { Expanded uncertainty of } \\
\text { vapor pressure at 95 \% level } \\
(\mathrm{Pa})\end{array}$ \\
\hline 333.28 & 15.8 & 1.5 \\
333.30 & 14.3 & 2.0 \\
343.22 & 31.7 & 1.8 \\
353.27 & 64.0 & 6.0 \\
363.25 & 122.3 & 2.8 \\
373.24 & 233 & 10 \\
383.28 & 401 & 24 \\
393.25 & 722 & 32 \\
403.26 & 1123 & 42 \\
413.16 & 1884 & 64 \\
\hline
\end{tabular}

${ }^{\mathrm{a}}$ Standard uncertainty of the temperature $\mathrm{u}(\mathrm{T})=0.04 \mathrm{~K}$ 
Table 18 Vapor pressure of liquid Dihydrolevoglucosenone (cyrene)

\begin{tabular}{lll}
\hline Temperature $\mathrm{K}^{\mathrm{a}}$ & $\begin{array}{l}\text { Vapor pressure } \\
\text { liquid }(\mathrm{Pa})\end{array}$ & $\begin{array}{l}\text { Expanded uncertainty of } \\
\text { vapor pressure at } 95 \% \text { level } \\
(\mathrm{Pa})\end{array}$ \\
\hline 298.26 & 14.4 & 2.4 \\
308.31 & 28.2 & 2.5 \\
318.24 & 51.5 & 8.0 \\
328.27 & 116.9 & 6.1 \\
333.32 & 157.3 & 7.0 \\
338.27 & 219.4 & 7.4 \\
343.26 & 294 & 15 \\
343.26 & 288 & 12 \\
353.25 & 506.1 & 7.1 \\
363.26 & 842 & 29 \\
373.24 & 1324 & 69 \\
378.23 & 1780 & 111 \\
383.26 & 2133 & 93 \\
393.18 & 3490 & 202 \\
403.16 & 5170 & 229 \\
\hline
\end{tabular}

*Standard uncertainty of the temperature $\mathrm{u}(\mathrm{T})=0.04 \mathrm{~K}$

Table 19 Vapor pressure of liquid Levoglucosenone

\begin{tabular}{lll}
\hline Temperature $(\mathrm{K})^{\mathrm{a}}$ & $\begin{array}{l}\text { Vapor pressure } \\
\text { liquid (Pa) }\end{array}$ & $\begin{array}{l}\text { Expanded uncertainty of } \\
\text { vapor pressure at 95 \% level } \\
(\mathrm{Pa})\end{array}$ \\
\hline 298.26 & 6.2 & 1.7 \\
303.25 & 10.0 & 2.3 \\
313.26 & 26.0 & 4.6 \\
323.25 & 42.2 & 7.8 \\
333.29 & 90.3 & 8.6 \\
343.24 & 205 & 11 \\
353.26 & 374 & 28 \\
363.27 & 621 & 36 \\
373.27 & 909 & 57 \\
383.29 & 1502 & 85 \\
393.26 & 2450 & 122 \\
403.30 & 3480 & 137 \\
\hline
\end{tabular}

${ }^{\text {a }}$ Standard uncertainty of the temperature $\mathrm{u}(\mathrm{T})=0.04 \mathrm{~K}$ 
Table 20 Vapor pressure of liquid $\gamma$-valerolactone

\begin{tabular}{lll}
\hline${\text { Temperature }(\mathrm{K})^{\mathrm{a}}}^{\mathrm{a}}$ & $\begin{array}{l}\text { Vapor pressure } \\
\text { liquid }(\mathrm{Pa})\end{array}$ & $\begin{array}{l}\text { Expanded uncertainty of } \\
\text { vapor pressure at } 95 \% \text { level } \\
(\mathrm{Pa})\end{array}$ \\
\hline 298.23 & 43.6 & 3.1 \\
303.20 & 64.8 & 3.2 \\
313.25 & 136.7 & 7.0 \\
323.25 & 244 & 12 \\
333.29 & 453 & 21 \\
343.18 & 763 & 22 \\
343.28 & 791 & 38 \\
353.26 & 1293 & 29 \\
363.25 & 2060 & 61 \\
373.17 & 3120 & 173 \\
383.12 & 2280 & 144 \\
393.08 & 3520 & 223 \\
403.15 & 5320 & 346 \\
\hline
\end{tabular}

${ }^{a}$ Standard uncertainty of the temperature $\mathrm{u}(\mathrm{T})=0.04 \mathrm{~K}$ 


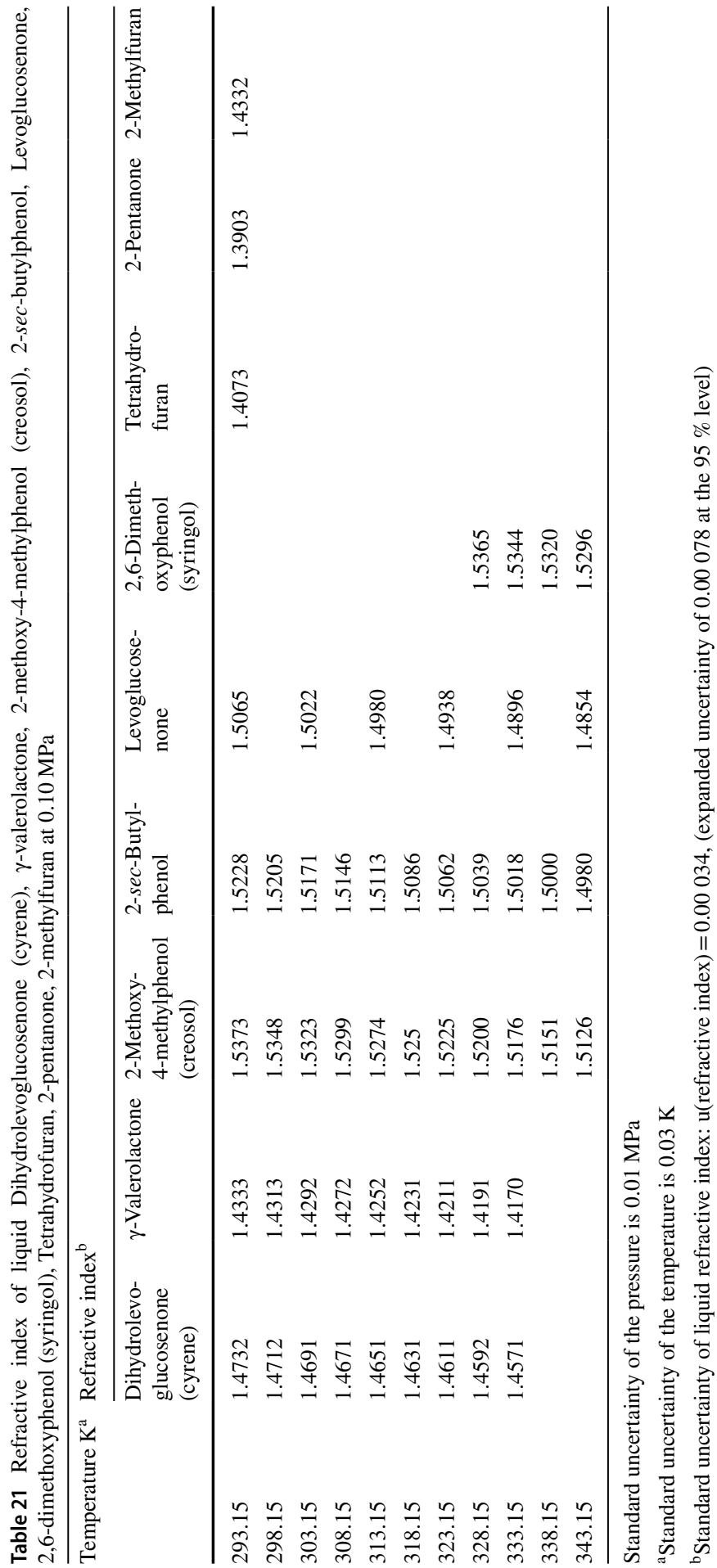


Table 22 Comparison of experimental density values from this study with literature values

\begin{tabular}{llll}
\hline Compound & \multicolumn{2}{l}{ Density at 293.15 K $\left(\mathrm{kg} \cdot \mathrm{m}^{-3}\right)$} & References \\
\cline { 2 - 3 } & This work & Literature $^{\mathrm{a}}$ & \\
\hline 2-Methoxy-4-methylphenol & $1096.6 \pm 1.3$ & 1090.05 & {$[76]$} \\
2-Methylfuran & $915.46 \pm 0.1$ & $915.31 \pm 0.48$ & {$[77-81]$} \\
2-Pentanone & $806.21 \pm 0.41$ & $807.80 \pm 0.56$ & {$[82-100]$} \\
2-sec-Butylphenol & $977.24 \pm 0.22^{\mathrm{b}}$ & $980.4^{\mathrm{b}}$ & {$[101]$} \\
2,6-Dimethoxyphenol & $1158.57 \pm 0.6^{\mathrm{c}}$ & - & \\
Cyclopentyl methyl ether & $862.95 \pm 0.2$ & 862.80 & {$[102]$} \\
& & 860.43 & {$[103]$} \\
Dihydrolevoglucosenone & $1250.75 \pm 0.51$ & 1250 & {$[3]$} \\
& & 1251.7 & {$[44]$} \\
Furfural & $1159.79 \pm 0.47$ & $1160.10 \pm 0.45$ & {$[79,93,104-113]$} \\
Levoglucosenone & $1303.6 \pm 3.4$ & - & {$[58,114-129]$} \\
Tetrahydrofuran & $887.47 \pm 0.2$ & $887.57 \pm 0.14$ & {$[56,67,68,130-132]$} \\
$\gamma$-Valerolactone & - & $1054.61 \pm 1.4$ & \\
\hline
\end{tabular}

${ }^{a}$ Mean of literature values, if more than two literature values were available and outliers were removed

${ }^{\mathrm{b}}$ At $298.15 \mathrm{~K}$

${ }^{\mathrm{c}}$ At $333.16 \mathrm{~K}$

Fig. 2 Comparison of the literature sources that give the density of tetrahydrofuran at elevated pressures. $\times$ This work, $-[58]$, [59], $[60], \triangle[61], \triangleleft[62]$, $\star[63],+[64]$

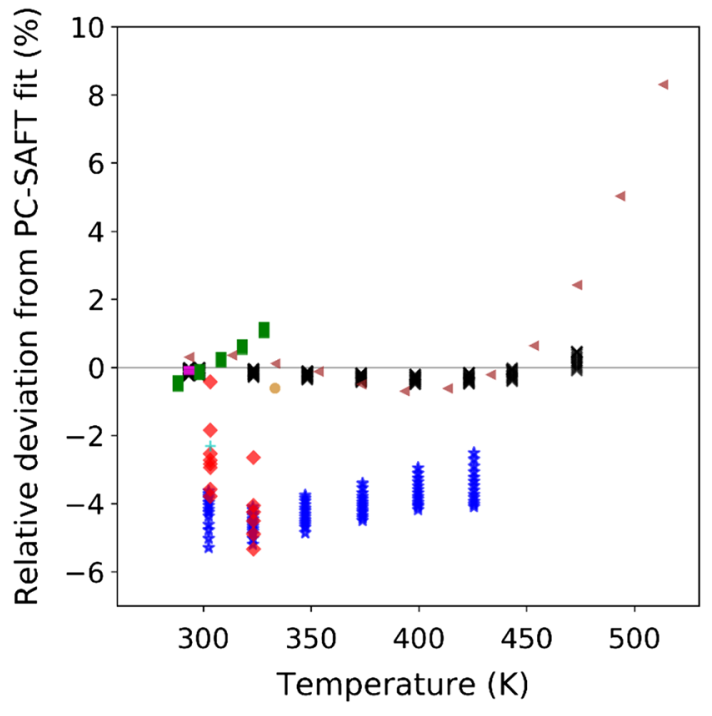




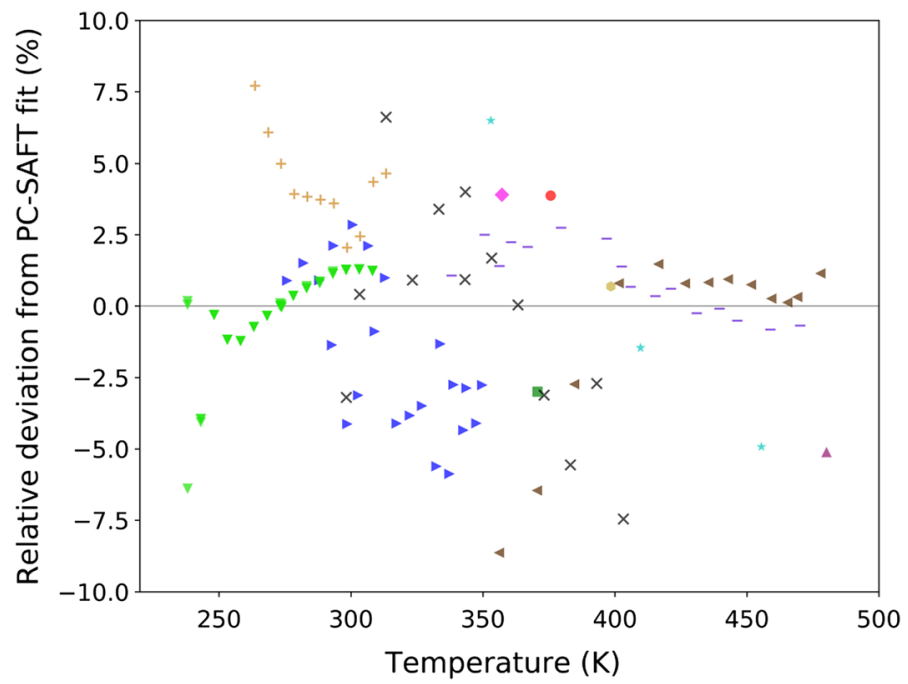

Fig. 3 Comparison of vapor pressure data for gamma-valerolactone from different sources. $\times$ This work, $\nabla[65],-[66], \backsim[67], \bullet[68],<[69], \triangleright[70], \triangle[56], \star[71],+[72],\lrcorner[73], \Delta[74]$

Table 23 Normal boiling points and enthalpies of vaporization of the bio-compounds

\begin{tabular}{|c|c|c|c|c|}
\hline \multirow[t]{2}{*}{ Compound } & \multicolumn{2}{|c|}{ Normal boiling point $^{1}(\mathrm{~K})$} & \multicolumn{2}{|c|}{$\begin{array}{l}\text { Enthalpy of vaporization }{ }^{2} \text { at } \\
298.15 \mathrm{~K}\left(\mathrm{~kJ} \cdot \mathrm{mol}^{-1}\right)\end{array}$} \\
\hline & This work & Literature $^{\mathrm{a}}$ & This work & Literature \\
\hline 2-Methoxy-4-methylphenol & 493.9 & 494.2 & 64.58 & $70.9^{\mathrm{a}}$ \\
\hline 2-Methylfuran & 337.4 & $337 \pm 1$ & 32.11 & $32.4^{\mathrm{b}}$ \\
\hline 2-Pentanone & 375.3 & $375 . \pm 1$ & 38.26 & $38.4^{\mathrm{a}}$ \\
\hline 2-sec-Butylphenol & 503.5 & $500 . \pm 4$ & 78.38 & NA \\
\hline 2,6-Dimethoxyphenol & 535.0 & $536 \pm 5$ & 76.69 & NA \\
\hline Cyclopentyl methyl ether & 378.6 & $377.9^{c}$ & 37.41 & NA \\
\hline Dihydrolevoglucosenone & 499 & $476^{\mathrm{d}}$ & 57.65 & NA \\
\hline Furfural & 434.1 & $434.7 \pm 0.4$ & 50.57 & $50.7 \pm 0.2$ \\
\hline Levoglucosenone & 504 & NA & 61.56 & NA \\
\hline Tetrahydrofuran & 339.2 & $339 . \pm 1$ & 31.87 & $32.16^{\mathrm{a}}$ \\
\hline$\gamma$-Valerolactone & 478.3 & 480.7 & 53.83 & $53.9 \pm 0.2^{\mathrm{e}}$ \\
\hline
\end{tabular}

All values were calculated using the PC-SAFT equation of state

NA value is not available at $298.15 \mathrm{~K}$

${ }^{\text {a}}$ Ref. [29]

${ }^{b}$ Data from Ref. [29] correlated to obtain value at $298.15 \mathrm{~K}$

${ }^{\mathrm{c}}$ Ref. [19] at pressure 99,800 $\mathrm{Pa}$

${ }^{\mathrm{d}}$ Ref. [3]

${ }^{\mathrm{e}}$ Ref. [70]

${ }^{1}$ At a pressure of $101,325 \mathrm{~Pa}$, standard uncertainty $\mathrm{u}$ (normal boiling point this work) $=1.5 \mathrm{~K}$

${ }^{2} \mathrm{u}($ enthalpy of vaporization $)=0.7 \mathrm{~kJ} \cdot \mathrm{mol}^{-1}$ 
Acknowledgements Open access funding provided by Aalto University. We would like to thank the company Circa for providing the dihydrolevoglucosenone and levoglucosenone samples.

Open Access This article is distributed under the terms of the Creative Commons Attribution 4.0 International License (http://creativecommons.org/licenses/by/4.0/), which permits unrestricted use, distribution, and reproduction in any medium, provided you give appropriate credit to the original author(s) and the source, provide a link to the Creative Commons license, and indicate if changes were made.

\section{References}

1. T. Werpy, G. Petersen, Top Value Added Chemicals from Biomass: Volume I-Results of Screening for Potential Candidates from Sugars and Synthesis Gas (DOE/GO-102004-1992, 15008859, 2004), http://www.osti.gov/servlets/purl/15008859/. Accessed: 01 Oct 2019

2. F.H. Isikgor, C.R. Becer, Polym. Chem. 6, 4497 (2015)

3. J. Sherwood, M. De Bruyn, A. Constantinou, L. Moity, R.C. McElroy, T.J. Farmer, T. Duncan, W. Raverty, A.J. Hunt, J.H. Clark, Chem. Commun. 50, 9650 (2014)

4. J. Zhang, G.B. White, M.D. Ryan, A.J. Hunt, M.J. Katz, A.C.S. Sustain, Chem. Eng. 4, 7186 (2016)

5. N. Montazeri, A.C. Oliveira, B.H. Himelbloom, M.B. Leigh, C.A. Crapo, Food Sci. Nutr. 1, 102 (2013)

6. J. A. Maga, "Contribution of Phenolic Compounds to Smoke Flavor," in Phenolic Compounds in Food and Their Effects on Health I, vol. 506, (American Chemical Society, 1992), p. 170. https:// doi.org/10.1021/bk-1992-0506.ch013. Accessed 27 Feb 2019

7. A. Effendi, H. Gerhauser, A.V. Bridgwater, Renew. Sustain. Energy Rev. 12, 2092 (2008)

8. G.A. Burdock, Fenaroli's Handbook of Flavor Ingredients (CRC Press, Boca Raton, 2016)

9. Y. Gu, F. Jérôme, Chem. Soc. Rev. 42, 9550 (2013)

10. D.M. Alonso, S.G. Wettstein, J.Q. Bond, T.W. Root, J.A. Dumesic, Chemsuschem 4, 1078 (2011)

11. I. O'Hara, S. Mundree, Sugarcane-based Biofuels and Bioproducts (Wiley, Hoboken, 2016)

12. K. Yan, Y. Yang, J. Chai, Y. Lu, Appl. Catal. B Environ. 179, 292 (2015)

13. H. Q. Lê, Wood biorefinery concept based on $\gamma$-valerolactone/water fractionation, (Aalto University, 2018) https://aaltodoc.aalto.fi:443/handle/123456789/34508. Accessed 06 Mar 2019

14. H.Q. Lê, Y. Ma, M. Borrega, H. Sixta, Green Chem. 18, 5466 (2016)

15. G. Pruckmayr, P. Dreyfuss, and M. P. Dreyfuss, Polyethers, Tetrahydrofuran and Oxetane Polymers in Kirk-Othmer Encyclopedia of Chemical Technology, (John Wiley \& Sons 2001) https ://onlinelibrary.wiley.com/doi/abs/10.1002/0471238961.2005201816182103.a01. Accessed 01 Oct 2019

16. H. Müller, Tetrahydrofuran, Ullmanns Encycl. Ind. Chem., (Wiley-VCH Verlag GmbH \& Co. KGaA 2002) https://onlinelibrary.wiley.com/doi/10.1002/14356007.a26_221.pub2. Accessed: 01-Oct-2019

17. C. Wang, H. Xu, R. Daniel, A. Ghafourian, J.M. Herreros, S. Shuai, X. Ma, Fuel 103, 200 (2013)

18. M. Männistö, Phase equilibria of aqueous bio-component mixtures with different solvents for the design of a liquid-liquid extraction unit, (Aalto University, 2018) https://aaltodoc.aalto.fi:443/handl e/123456789/35586 Accessed 01 Oct 2019

19. M. Männistö, J.-P. Pokki, V. Alopaeus, J. Chem. Thermodyn. 119, 61 (2018)

20. R. Mariscal, P. Maireles-Torres, M. Ojeda, I. Sádaba, M.L. Granados, Energy Environ. Sci. 9, 1144 (2016)

21. N.M. Bennett, S.S. Helle, S.J.B. Duff, Bioresour. Technol. 100, 6059 (2009)

22. L. Grazia, A. Lolli, F. Folco, Y. Zhang, S. Albonetti, F. Cavani, Catal. Sci. Technol. 6, 4418 (2016)

23. J.N. Murwanashyaka, H. Pakdel, C. Roy, Sep. Purif. Technol. 24, 155 (2001)

24. S. Kim, J. Chen, T. Cheng, A. Gindulyte, J. He, S. He, Q. Li, B.A. Shoemaker, P.A. Thiessen, B. Yu, L. Zaslavsky, J. Zhang, E.E. Bolton, Nucleic Acids Res. 47, D1102 (2019)

25. "Home - Finnish Meteorological Institute." https://en.ilmatieteenlaitos.fi/. Accessed: 01-Oct 2019

26. R. Span, E.W. Lemmon, R.T. Jacobsen, W. Wagner, A. Yokozeki, J. Phys. Chem. Ref. Data 29, $1361(2000)$ 
27. W. Wagner, A. Pruss, J. Phys. Chem. Ref. Data 31, 387 (2002)

28. I.H. Bell, J. Wronski, S. Quoilin, V. Lemort, Ind. Eng. Chem. Res. 53, 2498 (2014)

29. Thermophysical Properties of Fluid Systems. http://webbook.nist.gov/chemistry/fluid/. Accessed 01 Oct 2019

30. R. Storn, K. Price, J. Glob. Optim. 11, 341 (1997)

31. E. Jones, E. Oliphant, P. Peterson, SciPy: Open Source Scientific Tools for Python. 2001 http:// www.scipy.org/. Accessed 01 Oct 2019

32. OECD Guidelines for the Testing of Chemicals, Section 1. (OECD Publishing, 2006). https://www. oecd-ilibrary.org/environment/oecd-guidelines-for-the-testing-of-chemicals-section-1-physicalchemical-properties_20745753. Accessed 01 Oct 2019

33. S.P. Verevkin, D. Wandschneider, A. Heintz, J. Chem. Eng. Data 45, 618 (2000)

34. Evaluation of measurement data - Supplement 1 to the "Guide to the expression of uncertainty in measurement"-Propagation of distributions using a Monte Carlo method. 1st edn.(Joint Committee for Guides in Metrology, 2008). https://www.bipm.org/utils/common/documents/jcgm/ JCGM_101_2008_E.pdf Accessed 01 Oct 2019

35. P. Schiebener, J. Straub, J. M. H. Levelt Sengers, J. S. Gallagher, J. Phys. Chem. Ref. Data 19, 677 (1990)

36. J. Gross, G. Sadowski, Ind. Eng. Chem. Res. 40, 1244 (2001)

37. M. Kleiner, G. Sadowski, J. Phys. Chem. C 111, 15544 (2007)

38. J. Gross, J. Vrabec, AIChE J. 52, 1194 (2006)

39. J. Gross, G. Sadowski, Ind. Eng. Chem. Res. 41, 5510 (2002)

40. S.H. Huang, M. Radosz, Ind. Eng. Chem. Res. 29, 2284 (1990)

41. S.H. Huang, M. Radosz, Ind. Eng. Chem. Res. 30, 1994 (1991)

42. S.H. Huang, M. Radosz, Ind. Eng. Chem. Res. 32, 762 (1993)

43. A.J. de Villiers, C.E. Schwarz, A.J. Burger, Fluid Phase Equilib. 305, 174 (2011)

44. A. Misefari, "Investigation of the spectroscopic, chemical and physical properties of Cyrene and its hydrate," (University of York, 2017)

45. DIPPR Project 801-Full Version. Design Institute For Physical Properties. http://app.knovel.com/ hotlink/toc/id:kpDIPPRPF7/dippr-project-801-full/dippr-project-801-full. Accessed 01 Oct 2019

46. K. Watanabe, N. Yamagiwa, Y. Torisawa, Org. Process Res. Dev. 11, 251 (2007)

47. V. Makovskaya, J.R. Dean, W.R. Tomlinson, M. Comber, Anal. Chim. Acta 315, 193 (1995)

48. S.V. Anantakrishnan, S. Soundararajan, Proc. Indian Acad. Sci. Sect. A. 59, 365 (1964)

49. D.R. Lide, CRC Handbook of Chemistry and Physics, 84th edn. (CRC Press, Cleveland, 2004)

50. M.S. Qureshi, T. Le Nedelec, H. Guerrero-Amaya, P. Uusi-Kyyny, D. Richon, V. Alopaeus, J. Chem. Thermodyn. 105, 296 (2017)

51. N.M.E. Auger, I. Gedik, N. Ferrando, M. Dicko, P. Paricaud, F. Volle, J.P. Passarello, J.C. de Hemptinne, P. Tobaly, P. Stringari, C. Coquelet, D. Ramjugernath, P. Naidoo, R. Lugoc, Fluid Phase Equilib. 337, 234 (2013)

52. J. Wang, R. Lakerveld, AIChE J. 64, 1205 (2018)

53. K. Řehák, M. Klajmon, M. Strejc, P. Morávek, J. Chem. Eng. Data 62, 3878 (2017)

54. S. Jaatinen, J. Touronen, R. Karinen, P. Uusi-Kyyny, V. Alopaeus, J. Chem. Thermodyn. 112, 1 (2017)

55. E. Altuntepe, A. Reinhardt, J. Brinkmann, T. Briesemann, G. Sadowski, C. Held, J. Chem. Eng. Data 62, 1983 (2017)

56. M. Klajmon, K. Řehák, P. Morávek, M. Matoušová, J. Chem. Eng. Data 60, 1362 (2015)

57. T.A. Apaev, A.M. Kerimov, N.K. Dzhanakhmedov, Ukr. Fiz. ZhUkr. Ed. 22, 408 (1977)

58. K. Holzapfel, G. Goetze, F. Kohler, Int. Data Ser. Sel. Data Mix. Ser. A. 15, 263 (1987)

59. Y. Sato, H. Yoshioka, S. Aikawa, R.L. Smith, Int. J. Thermophys. 31, 1896 (2010)

60. U.P. Govender, T.M. Letcher, S.K. Garg, J.C.J. Ahluwalia, Chem. Eng. Data 41, 147 (1996)

61. I. Vasileva, A. Naumova, A. Polyakov, T. Tyvina, N. Kozlova, Zh. Prikl. Khim. 63, 1879 (1990)

62. L.G. Schornack, C.A. Eckert, J. Phys. Chem. 74, 3014 (1970)

63. W. Zhang, E. Kiran, J. Chem. Thermodyn. 35, 605 (2003)

64. S.D. Hamann, F. Smith, Aust. J. Chem. 24, 2431 (1971)

65. V. Pokorný, V. Štejfa, M. Fulem, C. Červinka, K. Růžička, J. Chem. Eng. Data 62, 4174 (2017)

66. A. Zaitseva, J.-P. Pokki, H.Q. Le, V. Alopaeus, H. Sixta, J. Chem. Eng. Data 61, 881 (2016)

67. B.B. Allen, B.W. Wyatt, H.R. Henze, J. Am. Chem. Soc. 61, 843 (1939)

68. E.J. Boorman, R.P. Linstead, J. Chem. Soc. Lond. 577, 580 (1933)

69. D. Havasi, P. Mizsey, L.T. Mika, J. Chem. Eng. Data 61, 1502 (2016) 
70. V.N. Emel'yanenko, S.A. Kozlova, S.P. Verevkin, G.N. Roganov, J. Chem. Thermodyn. 40, 911 (2008)

71. W. Huckel, W. Gelmroth, Liebigs Ann. Chem. 514, 233 (1934)

72. R.H. Leonard, Ind. Eng. Chem. 48, 1330 (1956)

73. H.A. Schuette, R.W. Thomas, J. Am. Chem. Soc. 52, 3010 (1930)

74. A.R. Ubbelohde, Proc. R. Soc. Lond. Ser. Math. Phys. Sci. 152, 378 (1935)

75. Z.S. Baird, A. Dahlberg, P. Uusi-Kyyny, N. Osmanbegovic, J. Witos, J. Helminen, D. Cederkrantz, P. Hyväri, V. Alopaeus, I. Kilpeläinen, S.K. Wiedmer, H. Sixta, Int. J. Thermophys. 40, 71 (2019)

76. W.H. Perkin, J. Chem. Soc. Trans. 69, 1025 (1896)

77. S. Jężak, M. Dzida, M. Zorębski, Fuel 184, 334 (2016)

78. L. Lomba, I. Aznar, I. Gascón, C. Lafuente, B. Giner, Thermochim. Acta 617, 54 (2015)

79. R.F. Holdren, R.M. Hixon, Ind. Eng. Chem. 38, 1061 (1946)

80. A.S. Smith, J.F. LaBonte, Ind. Eng. Chem. 44, 2740 (1952)

81. S. Loras, A. Aucejo, J.B. Montón, J. Wisniak, H. Segura, J. Chem. Eng. Data 47, 1256 (2002)

82. R.H. Cole, J. Chem. Phys. 9, 251 (1941)

83. N. C. Cook, Final Rep. Stand. Proj. on Oxygenated Compounds, (Penn. State Univ., College Park, PA, USA, 1952), https://webbook.nist.gov/cgi/cbook.cgi?ID=C589822\&Mask=FFF\#ref-3. Accessed 03 Oct 2019

84. D. M. Cowan, G. H. Jeffery, A. I. Vogel, Chem. Soc. Resumed 171 (1940)

85. A.K. Doolittle, The technology of solvents and plasticizers (Wiley, New York, 1954)

86. F. Eisenlohr, Z Phys. Chem. 75, 585 (1910)

87. M. Favorsky, M. Tchitchonkine, I. Iwanow, C.R. Hebd, Seances. Acad. Sci. 199, 1229 (1934)

88. R. Gartenmeister, Z. Phys, Chem. Stoechiom. Verwandtschaft 6, 524 (1890)

89. N. I. Shuikin, R. A. Karakhanov, Ibrakhimov, Izv. Akad. Nauk. SSSR Ser. Khim. 165, (1965)

90. T. Tomonari, Z. Phys, Chem. Abt. B 32, 202 (1936)

91. F.J. Wright, J. Chem. Eng. Data 6, 454 (1961)

92. 1991 TRC-Thermodynamic Tables-Non Hydrocarbons, (Thermodynamics Research Center. College Station, TX, USA: The Texas A \& M University System, 1991)

93. J.A. Riddick, W.B. Bunger, T.K. Sakano, Organic Solvents: Physical Properties and Methods of Purification, 4th edn. (Wiley-Interscience, New York, 1986)

94. Selected Values of Properties of Chemical Compounds 1980-extant Data Project, Thermodynamics Research Center, Texas A\&M University, College Station, Texas, USA

95. J. Timmermans, Physico-chemical Constants of Pure Organic Compounds (Elsevier, New York, 1965)

96. D.L. Gonzalez, P.D. Ting, G.J. Hirasaki, W.G. Chapman, Energy Fuels 19, 1230 (2005)

97. B. Gonzalez, A. Domínguez, J. Tojo, J. Chem. Thermodyn. 38, 707 (2006)

98. A. Nikolić, B. Jović, M. Vraneš, S. Dožić, S. Gadžurić, J. Chem. Eng. Data 58, 1070 (2013)

99. W.A. Scheller, S.V.N. Rao, J. Chem. Eng. Data 18, 223 (1973)

100. A.P. Toropov, Zh. Obshch. Khim. 26, 1285 (1956)

101. F. Hawthorne, D.J. Cram, J. Am. Chem. Soc. 74, 5859 (1952)

102. H. Zhang, J. Chem. Eng. Data 60, 1371 (2015)

103. A. Randová, O. Vopička, L. Bartovská, K. Friess, Chem. Pap. 72, 947 (2018)

104. J. G. M. Bremner, R. K. F. Keeys, J. Chem. Soc. Resumed 1068 (1947)

105. F.H. Garner, R.T.W. Hall, J. Inst. Pet. 41, 1 (1955)

106. Y.A. Tsirlin, Zh. Fiz. Khim. 36, 1673 (1962)

107. Y.A. Tsirlin, V.A. Vasil'eva, Zh. Prikl. Khim. Leningr. 44, 1121 (1971)

108. A.Y. Karmilchik, L.S. Efimova, Zh. Fiz. Khim. 42, 2305 (1968)

109. S.E. Kharin, V.M. Perelygin, K.K. Polyanskii, Gidroliz. Lesokhim. Prom-St. 23, 10 (1970)

110. P. Landrieu, F. Baylocq, J.R. Johnson, Bull. Soc. Chim. Fr. 45, 36 (1929)

111. G. Mains, Chem. Met. Eng. 26, 779 (1922)

112. V.P. Belousov, S.G. Shutin, Z. Phys, Chem. Leipz. 269, 689 (1988)

113. J. Staroske, G. Figurski, Chem. Tech. Leipz. 44, 64 (1992)

114. D. Papoušek, L. Págo, Collect. Czechoslov. Chem. Commun. 24, 2666 (1959)

115. A. G. Demakhin, S. M. Ponomarenko, O. Y. Yudina, Oniitekhim 1, (1991)

116. J. Lampa, J. Matous, J.P. Novak, J. Pick, Collect. Czech. Chem. Commun. 45, 1159 (1980)

117. V.A. Shnitko, V.B. Kogan, T.V. Sheblom, Zh. Prikl. Khim. Leningr. 42, 2389 (1969)

118. C. E. Boord, K. W. Greenlee, W. L. Perilstein, Ohio State Univ., Am. Pet. Inst. Res. Proj. 45, Eighth Annu. Rep., (1946) 
119. Tetrahydrofuran technical data. BASF Wyandotte Corporation, Parsippany, New Jersey

120. K.I. Areshidze, E.K. Tavartkiladze, G.O. Chivadze, J. Appl. Chem. USSR Engl. Transl. 43, 606 (1970)

121. T.B. Du, M. Tang, Y.-P. Chen, Fluid Phase Equilib. 192, 71 (2001)

122. M. Geppert-Rybczyńska, A. Heintz, J.K. Lehmann, A. Golus, J. Chem. Eng. Data 55, 4114 (2010)

123. M. Geppert-Rybczyńska, M. Sitarek, J. Chem. Eng. Data 59, 1213 (2014)

124. E.V. Ivanov, J. Chem. Thermodyn. 72, 37 (2014)

125. Á. Piñeiro, P. Brocos, A. Amigo, M. Pintos, R. Bravo, J. Solut. Chem. 31, 369 (2002)

126. F. Ratkovics, M.L. Parragi, Magy. Kem. Foly. 90, 28 (1984)

127. R.B. Torres, M.I. Ortolan, P.L.O. Volpe, J. Chem. Thermodyn. 40, 442 (2008)

128. D. Zikmundova, J. Matouš, J.P. Novak, V. Kubíček, J. Pick, Fluid Phase Equilib. 54, 93 (1990)

129. E.M. Živković, D.M. Bajić, I.R. Radović, S.P. Šerbanović, M.L. Kijevčanin, Fluid Phase Equilib. 373, 1 (2014)

130. M.L.P. Leitão, G. Pilcher, Y. Meng-Yan, J.M. Brown, A.D. Conn, J. Chem. Thermodyn. 22, 885 (1990)

131. 1998-89 Handbook of Fine Chemical Handbook of Fine Chemicals. Milwaukee, WI, USA: Aldrich Chemical Co

132. U.M. Fornefeld-Schwarz, P. Svejda, J. Chem. Eng. Data 44, 597 (1999)

Publisher's Note Springer Nature remains neutral with regard to jurisdictional claims in published maps and institutional affiliations. 